\title{
Artificial neural networks prediction of in-plane and out-of-plane homogenized coefficients of hollow blocks masonry wall
}

\author{
Houda FRIAA ${ }^{1 *}$, Myriam LAROUSSI HELLARA ${ }^{1}$, Ioannis STEFANOU ${ }^{2}$, Karam SAB $^{2}$, \\ Abdelwaheb DOGUI ${ }^{1}$ \\ 1 University of Monastir, National Engineering School of Monastir, Mechanical Engineering \\ Laboratory, LR99ES32, 5019, Monastir, Tunisia \\ 2 Université Paris-Est, Laboratoire Navier UMR 8205/CERMES, École des Ponts ParisTech, \\ IFSTTAR, CNRS, 77455 Marne-la-Vallée Cedex, France. \\ *Corresponding author. E-mail: houda.frea@ hotmail.com
}

\begin{abstract}
A masonry wall is a composite structure characterized by a large variety in geometrical and material parameters. The determination of the effective macroscopic properties, through the homogenization scheme, depends on a great number of variables. Thus, in order to replace heavy numerical simulation, in this paper, the use of artificial neural networks $(A N N)$ is proposed to predict elastic membrane and bending constants of the equivalent Love-Kirchhoff plate of hollow concrete blocks masonry wall. To model the $A N N$, a numerical periodic homogenization in several parameters is used. To construct the model, five main material and geometrical input parameters are utilized. Multilayer perceptron neural networks are designed and trained (with the best selected ANN model) by the sets of input-output patterns using the backpropagation algorithm. As a result, in both training and testing phases, the developed $A N N$ indicates high accuracy and precision in predicting the equivalent plate of a hollow masonry wall with insignificant error rates compared to FEM results.
\end{abstract}

Keywords: Artificial neural networks (ANN), Back-propagation, Orthotropic Love-Kirchhoff plate, Hollow blocks masonry, In-plane and out-of-plane loadings, Periodic numerical homogenization, Equivalent elastic properties, Influence of bond. 


\section{Introduction}

The masonry wall represents a composite structure obtained by a regular or irregular repetition of bricks or blocks joined by mortar, which is characterized by a large variety of arrangements, material, and geometrical parameters. Thus, the study of its anisotropy and its inhomogeneity behavior represents a challenging task. Furthermore, the prediction of the average mechanical properties of the structure is difficult, in particular for the elastic behavior. Actually, this average behavior strongly depends on the properties of the constituent materials. Sophisticated methods are then needed to derive the equivalent behavior. In addition, results for one masonry may differ from one another. Therefore, the development of an intelligent tool for the determination of equivalent orthotropic behavior of a large variety of masonry structures based on their geometrical and material properties provides a powerful tool for further studies. In the literature, several numerical and analytical models have been developed to study masonry structures using different methods.

A large number of micro-modelling and macro-modelling approaches have been proposed both in linear and nonlinear analyses of masonry structure [1-3]. In the former, the overall masonry wall is regarded as a continuum equivalent homogeneous structure without considering any distinction between mortar joints and units [4] which makes it suitable for the analysis of complex and large structures due to its low computational cost. In the latter approach, the micro-modelling, masonry wall is characterized by a discretization at the level of units and joints [5]. Midway between these two approaches, there is the computational homogenization method. The use of such a method has been successfully implemented to derive the elastic behavior $[6,7]$ and the strength properties $[8-$ 10]. Generally, the macroscopic characterization is strongly connected to structure geometry (the texture), as well as units and joints mechanical behaviour. In the literature when dealing with the regular arrangement, the most investigated case using the homogenization method is the running bond pattern [11-13]. Recently, different periodic textures have been studied [14], as for instance English bond [15] or herringbone bond masonry [16]. Dealing with an irregular pattern, based on homogenization concepts, the effective properties in the elastic field was estimated [17-20], also the strength domain was estimated [9][21-23].

Nevertheless, very limited analytical and numerical models have been proposed to derive the equivalent behaviour of masonry structure on parametric analysis. Using a finite-element technique, Cecchi and Di Marco [24] carried out a parametric study on the ratio between the mechanical characteristics of the blocks and of the joints, defining the ultimate behavior when considering the block as infinitely stiff. Following this work, Cecchi et al. [6] presented a study dealing with the determination of an analytical expression of the macroscopic overall effective elastic properties of masonry considering three intrinsic disruptive parameters. Furthermore, Drougkas et al. [25] 
presented an analytical model for the analysis of masonry periodic unit cells based on detailed micromodeling. However, these suggested methods may require high computational costs since the formulation of equivalent models is complex and requires a huge number of simulations. Consequently, it seems interesting to use a faster method which reduces the complexity of this study. One of the most popular and efficient methods is the use of Artificial Neural Networks (ANNs). The latter represents powerful data modeling tools, which are able to predict, classify and represent complex nonlinear relationships between independent variables and responses. They have been recently employed to solve a variety of civil engineering problems [26-28]. Several researchers have successfully used ANNs to estimate the compressive strength and elastic modulus of different types of concrete [29, 30]. Added to that, some studies have focused on cement mortar and the influence of element strength on compressive mortar strength [31]. To predict and model the compressive strength of recycled concrete aggregates, both the ANNs and the responses surface methodology (RSM) were used in [32], as a result, both approaches correlate very well with the experimental data. However, the ANN model showed better accuracy.

During the last decades, very few studies have incorporated the use of ANNs for the prediction of masonry behavior in general. As an example, ANN was used to approximate the masonry structure failure surface under biaxial compressive stress [33]. Furthermore, new studies have been conducted on the prediction of masonry behavior using ANN. Asteris et al.[28] put in evidence the ability of ANN to solve nonlinear problems by modeling an anisotropy masonry failure criterion under biaxial compressive strength. Zhou et al. [34] used ANNs and adaptive neuro-fuzzy networks to predict the shear resistance of fully grouted reinforced concrete masonry. Zhang et al. [35] developed an ANN model for predicting the cracking patterns of different masonry wallettes subjected to a vertical load. Recently, ANN combined with artificial bee colony [36] was presented for solving problems related to the safety of retaining walls in geotechnics. In another study, Garzón-Roca et al.[37] adopted ANN to predict the maximum axial load capacity of masonry structure, as well as adopted ANNs and Fuzzy Logic in [38] to estimate the compressive strength of brick masonry structure. Furthermore, optimized ANN with PSO (particle swarm optimization) was successfully used to predict the seismic response of a short structure in [39]. Zhou et al. [40] proposed the use of an artificial intelligence method to predict the failure of a laterally loaded masonry wall based on the experimental analysis. Three ANN models were trained and adjusted to optimize its parameters.

The results of previous studies have shown the power of statistical methods to establish a relationship between critical parameters and complex behavior systems. However, no research has been conducted on the application of the well-known artificial intelligent technique to estimate the macroscopic equivalent elastic properties of the masonry structure. The current study investigates 
for the first time the development of the ANN model to predict the properties of the equivalent LoveKirchhoff plate of a hollow blocks masonry wall mainly based on five material and geometrical parameters. Subsequently, the relationship between responses and different factors is analyzed. Additionally, the estimation of capabilities and modeling efficiencies of ANN models are statistically proved, using various parameters, such as the coefficient of determination $\left(R^{2}\right)$, the mean absolute percentage error (MAPE), and the root mean square error (RMSE).

\section{Masonry in-plane and out-of-plane homogenization scheme: influence of bond}

\subsection{Geometry description}

Numerical homogenization is applied in this paper to estimate the equivalent behaviour of the periodic masonry assemblage, by taking into account the characteristics of different constituents and by focusing on the influence of different bond configurations. Indeed, the units and mortar relative arrangement have a significant influence on the equivalent elastic properties. Although, the research when dealing with the influence of the masonry bond pattern appears still somewhat fragmented even if some researches have been proposed [14][41]. It has been demonstrated that significant changes occur in the response in terms of stiffness, strength....

In what follows to better clarify how the equivalent elastic behaviour is depending on the texture, special attention is paid to the influence of bond pattern by studying several commonly used periodic arrangements masonry walls.

In this paper, we consider a running bond, a stack bond and an English bond masonry structures denoted by $M$ with height $H$ and length $L$. These walls are formed by periodically arranged hollow concrete blocks, between which bed and head mortar are laid with a thickness of $e^{v}$ and $e^{h}$, respectively as depicted in Fig.1. The used units are the most common construction materials, known by classification B40 in accordance with NF P 14-402 [42]. The geometric parameters of the latter are: $($ length $=500 \mathrm{~mm} \times$ height $=200 \mathrm{~mm} \times$ thickness $=200 \mathrm{~mm}$ ). Due to their low cost and their durability, they are widely used in masonry structures all over the world such as structural elements of large commercial and government buildings like schools, office buildings, and hospitals. Concrete blocks are also fireproofing and resist moisture, making it ideal for wet environments, such as a house near the ocean or in a basement. To study the behaviour of such a complex structure subjected to in-plane and out-of-plane loads by means of Finite Element Modelling (FEM), a very fine mesh is needed. Hence, to optimize the computational cost, it is convenient to look for an equivalent plate model for this 3D structure by means of the periodic homogenization scheme. 
The considered walls are regarded as periodic structures in their plane as shown in Fig.1. Therefore, thanks to the periodicity of the building arrangement within in-plane directions $\left(x_{1}, x_{2}\right)$, each entire wall can be considered as a repetition of a basic cell called the unit-cell and denoted by $Y$. The choice of this basic cell depends strongly on the geometry of the masonry structure. For the abovedescribed structures, the chosen basic cells for running bond, stack bond, and English bond pattern respectively are shown in Fig.2. These chosen unit-cells own all the necessary information to describe the geometry of the whole structures M. For the running bond pattern, the unit-cell is formed by the equivalent of two hollow blocks rigidly connected by mortar, as illustrated in Fig.2(a). For the stack bond pattern, the unit-cell is formed by the equivalent of one hollow blocks surrounded by half mortar, as illustrated in Fig.2(b) and for the English bond the unit cell is formed by the equivalent of six blocks with different height joined with mortar. Let $x=\left[x_{1}, x_{1}\right]$ be the reference frame for the global description of the macroscopic structures (a whole wall), and the unit-cells reference frame is $\left[\underline{e}_{1}, \underline{e}_{2}, \underline{e}_{3}\right]$ which is associated with the local coordinate $y=\left[y_{1}, y_{2}, y_{3}\right]$. These unit-cells can be written as $Y=\omega \times]-\frac{t}{2} ; \frac{t}{2}\left[\right.$ where $\omega \in \mathbf{R}^{2}$ is their middle planes. The unit-cells boundary surface $\partial Y$ is given by $\partial Y=\partial Y_{l}^{ \pm} \cup \partial Y_{3}^{+} \cup \partial Y_{3}^{-}$where $\partial Y_{3}^{ \pm}=\omega \times[ \pm t / 2]$ and $l=1,2$ as shown in Fig.2. To be noted the same notation are adopted for different studied structures.

Fig.1. Periodic masonry walls understudy

Fig.2. Chosen unit-cells for masonry walls (a) running bond wall (b) stack bond wall (c) English bond wall

The geometrical parameters of the considered unit-cells are summarized in Table.1.

Table 1. Chosen unit-cells geometrical properties

\subsection{Homogenization scheme background}

In brief, the homogenization method represents a convenient approach to study masonry structures, both in the linear and non-linear range. It allows establishing constitutive relations in terms of macro or average stresses and strains from the microstructure and the constitutive relations of the individual 


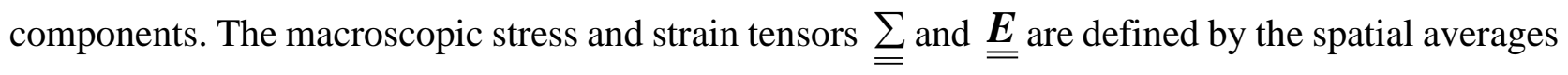
of the local stress and strain fields over the unit-cell $Y$ of volume V. Indeed, $\underline{=}=\langle\sigma\rangle=\frac{1}{V} \int_{Y} \underline{\sigma} d v$ and $\underline{\underline{E}}=\langle\varepsilon\rangle=\frac{1}{V} \int_{Y} \underline{\underline{\varepsilon}}(u) d v$; where $\underline{\underline{\varepsilon}}$ represents the local strain value which is directly dependent on the displacement field $\underline{\boldsymbol{u}}, \underline{\underline{\sigma}}$ is the local stress value and $\langle$.$\rangle is the average operator.$

It is noted that the wall length is much larger than the one size of the unit then it can be considered as a Love-Kirchhoff plate as it was demonstrated by Caillerie [43]. This plate occupies a domain $\Gamma \times]-\frac{t}{2}, \frac{t}{2}\left[\right.$ where $\Gamma \subset \mathbf{R}^{2}$ represents the middle surface and $t$ is its thickness.

Therefore, according to [7] and [44], the homogenization problem in the linear elastic range, in presence of coupled flexural and membrane loads (in-plane and out-of-plane loads), and under the assumption of the Love-Kirchhoff plate theory, can be written as follows:

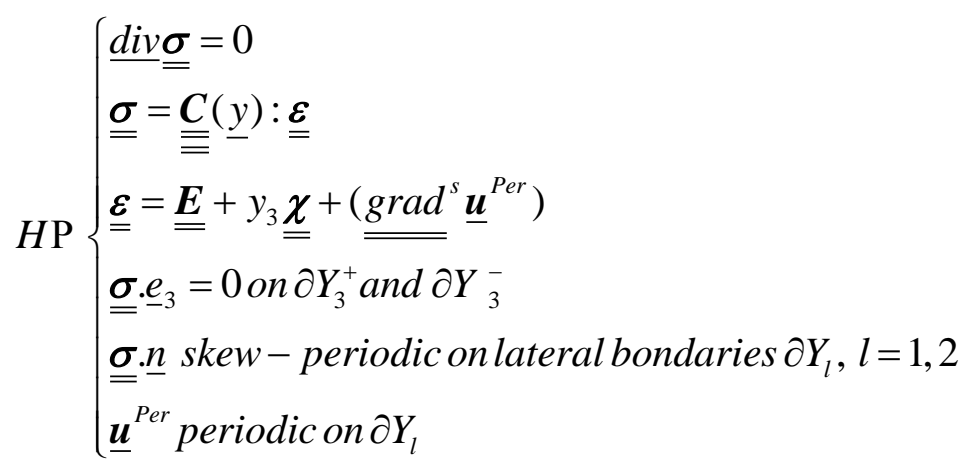

In the previous problem $(H P)$ : equation (1a) denotes the micro-equilibrium for the unit-cell with zero body forces, where $\underline{\underline{\text { div }}}$ is the divergence operator and $\underline{\underline{\sigma}}$ is the microscopic Cauchy stress tensor. Furthermore, equation (1b) represents the linear elastic behaviour law, where $\underline{\underline{\underline{\underline{C}}}}$ is the fourth-order elasticity compliance tensor, and the double contraction product is defined as $\underline{\underline{X}}: \underline{\underline{Y}}=X_{i j} Y_{j i}$. In equation (1c), the micro-strain tensor $\underline{\underline{\varepsilon}}$ is obtained as a linear combination among the macroscopic $\underline{\underline{E}}$ and $\underline{\underline{\chi}}$ tensors as well as a periodic strain field, where $\underline{\underline{E}}$ is the macroscopic in-plane strain tensor, $\underline{\underline{\chi \chi}}$ is the out-of-plane strain tensor, ${\underline{\underline{\mathrm{gad}^{s}}}}^{\text {is }}$ the symmetric part of the gradient operator and $\underline{\boldsymbol{u}}^{\text {per }}$ is the 2D periodic displacement vector. In equation (1e), $\underline{\boldsymbol{n}}$ represents the outer normal to the boundary of the unit-cell. The macroscopic tensors are related to the macroscopic displacement field components $U_{1}\left(x_{1}, x_{2}\right), U_{2}\left(x_{1}, x_{2}\right)$ and $U_{3}\left(x_{1}, x_{2}\right)$ by means of the classic 
relations $\quad E_{\alpha \beta}=1 / 2\left(U_{\alpha, \beta}+U_{\beta, \alpha}\right) \quad$ with $\quad E_{i 3}=0, \quad \chi_{\alpha \beta}=-U_{3, \alpha \beta} \quad$ and $\quad \chi_{i 3}=0$, with $\alpha, \beta=1,2$ and $i=1,2,3$.

The constitutive law of the macroscopic homogenized Love-Kirchhoff plate subjected to in-plane and out-of-plane loads may be written as:

$$
\begin{aligned}
& \underline{\underline{N}}=\langle\sigma\rangle=\underline{\underline{\underline{A}}}: \underline{\underline{E}}+\underline{\underline{\underline{B}}}: \underline{\underline{\chi}} \\
& \underline{\underline{\boldsymbol{M}}}=\left\langle y_{3} \sigma\right\rangle={ }^{t} \underline{\underline{\underline{B}}}: \underline{\underline{E}}+\underline{\underline{\underline{D}}: \underline{\chi}}
\end{aligned}
$$

where $\underline{\underline{N}}=\left(N_{\alpha \beta}\right)$ is the macroscopic in-plane stress field and $\underline{\underline{M}}=\left(M_{\alpha \beta}\right)$ is the macroscopic outof-plane stress field. Note that both $\underline{\underline{N}}$ and $\underline{\underline{M}}$ follow the symmetry of stress tensor. Moreover, $\underline{\underline{\underline{A}}}$ , $\underline{\underline{\underline{B}}}$ and $\underline{\underline{\underline{\boldsymbol{D}}}}$ are the constitute homogenized plates tensors. It is noted that the membrane and bending constants can be obtained by solving boundary value problems on the unit-cell.

The problem $(H P)$ basically imposes the membrane strain $\underline{\underline{E}}$ and curvature $\underline{\underline{\chi}}$ on average on the chosen unit-cells while taking into account periodicity in the plane $\left(y_{1}, y_{2}\right)$ of the structures and traction free conditions on the upper and lower faces of the plate [45], where $\underline{\underline{E}}$ and $\underline{\underline{\chi}}$ are defined as:

$\underline{\underline{\boldsymbol{E}}}=\left(\begin{array}{ccc}E_{11} & E_{12} & 0 \\ E_{21} & E_{22} & 0 \\ 0 & 0 & 0\end{array}\right)$ and $\underline{\underline{\chi}}=\left(\begin{array}{ccc}\chi_{11} & \chi_{12} & 0 \\ \chi_{21} & \chi_{22} & 0 \\ 0 & 0 & 0\end{array}\right)$

In homogenization, it is known that the macroscopic field $(\underline{\underline{N}}, \underline{\underline{M}})$ and their associated stress-energy density varies slowly respecting the size of the unit-cell. Then, to obtain the Love-Kirchhoff stiffness tensors, the strain energy approach proposed by [46] is adopted. Using the Hill-Mandel principle, the average of the local energy in the unit-cell (microscopic energy) is equal to the whole plate energy (macroscopic energy) [45]. The plate strain energy density $W^{\text {homog }}$ is given by:

$$
W^{\text {homog }}(\underline{\underline{\boldsymbol{E}}}, \underline{\underline{\underline{\chi}}})=\frac{1}{2}(\underline{\underline{\boldsymbol{E}}}: \underline{\underline{\underline{\boldsymbol{A}}}}: \underline{\underline{\boldsymbol{E}}}+2 \underline{\underline{\boldsymbol{E}}}:(\underline{\underline{\underline{B}}}: \underline{\underline{\chi \chi \chi}})+\underline{\underline{\chi}}: \underline{\underline{\underline{\boldsymbol{D}}}}: \underline{\underline{\chi \chi \chi}})
$$

\subsection{Finite element derivation of in-plane and out-of-plane homogenized coefficients}

In this section, the homogenized membrane and bending constants of the equivalent plates of the studied structures are numerically determined by means of FEM. Anthoine [7] was the first to propose the use of standard FEM codes for solving the homogenization problem. In the following, 
the problem $(H P)$ is numerically solved in order to obtain the elastic strain energy of the chosen unit-cells. The three studied unit-cells have three planes of symmetry, as depicted in Fig.2. To reduce the computational cost, it is possible to solve the problem through the so-called "displacement method [47]" by considering only one-eighth of these unit-cells and by imposing specific boundary conditions. In this case, the constitutive homogenized plate tensor $\underline{\underline{\underline{\boldsymbol{B}}}}=\boldsymbol{O}$, whereas the symmetries lead to the following simplifications of the constitutive homogenized plate in Eqs. (2) and (3) [48] as follow:

$$
\begin{aligned}
& \left\{\begin{array}{l}
\boldsymbol{N}_{11}=A_{1111} E_{11}+A_{1122} E_{22} \\
\boldsymbol{N}_{22}=A_{1122} E_{11}+A_{2222} E_{22} \\
\boldsymbol{N}_{12}=2 A_{1212} E_{12}
\end{array}\right. \\
& \left\{\begin{array}{l}
M_{11}=D_{1111} \chi_{11}+D_{1122} \chi_{22} \\
M_{22}=D_{1122} \chi_{11}+D_{2222} \chi_{22} \\
M_{12}=2 D_{1212} \chi_{12}
\end{array}\right.
\end{aligned}
$$

It is noticeable that the elastostatic solution of the periodically arranged heterogenous structure must fulfil the average and the periodicity conditions on the strains (the same conditions for all the considered unit-cells: the three chosen unit cells have the same translation vectors to describe the considered periodic textures). For the previous problem, according to Anthoine [7], a strain periodic displacement may be written as:

$$
\underline{\boldsymbol{u}}=\left(\begin{array}{l}
\boldsymbol{E}_{11} y_{1}+\boldsymbol{E}_{12} y_{2}+y_{3}\left(\chi_{11} y_{1}+\chi_{12} y_{2}\right)+u_{1}^{P e r} \\
\boldsymbol{E}_{12} y_{1}+\boldsymbol{E}_{22} y_{2}+y_{3}\left(\chi_{12} y_{1}+\chi_{22} y_{2}\right)+u_{2}^{P e r} \\
-\left(1 / 2 \chi_{11} y_{1}^{2}+\chi_{12} y_{1} y_{2}+1 / 2 \chi_{22} y_{2}^{2}\right)+u_{3}^{P e r}
\end{array}\right)
$$

It is noted that the homogenized $A_{i j k l}$ and $D_{i j k l}$ moduli can be computed by separately applying three relevant cases for each one [44]:

$$
I_{E \chi}^{11}=\left[\begin{array}{ll}
1 & 0 \\
0 & 0
\end{array}\right], I_{E \chi}^{22}=\left[\begin{array}{ll}
0 & 0 \\
0 & 1
\end{array}\right], I_{E \chi}^{12}=\left[\begin{array}{ll}
0 & 1 \\
1 & 0
\end{array}\right]
$$

where $\underline{=}$ is the identity for in-plane elasticity with synthetically $\left(I_{E \chi}^{k l}\right)_{i j}=1 / 2\left(\delta_{i k} \delta_{j l}+\delta_{i l} \delta_{j k}\right)$, and $\delta_{i k}$ is the Krönecker symbol ( $\delta_{i k}=1$ if $i=k, \delta_{i k}=0$ otherwise).

Due to the symmetry of the chosen unit-cells, the periodic boundary conditions required in general cases are simplified by symmetric or skew-symmetric boundary conditions on lateral faces. The detailed prescribed displacements corresponding to membrane and curvature fields are summarized respectively in Fig.3(a) and Fig.3(b). It is worth noting that a home-made Python script coupled with ABAQUS is developed for this study to automatically program those periodic boundary conditions, 
the post-processing stages and the derivation of the homogenized coefficients as done by [49] for the numerical periodic homogenization to obtain an equivalent model.

Fig.3. Applied boundary conditions on one-eighth unit-cells (a) for symmetric loading, (b) for skew-symmetric loading

The FEM software ABAQUS Standard is used to solve the previous problem $(H P)$. Tetrahedral quadratic elements (C3D10) are chosen for both blocks and mortar (for all considered unit cells). The convergence study, not described here, has led to a mesh with three layers of elements in the thickness of the joints, resulting in a total number of 124,567 finite elements for the running bond pattern structure, in a total number of 76,614 finite elements for the stack bond pattern structure and in a total number of 323,140 finite elements for the English bond pattern structure.

The two constitutive materials, the concrete blocks and the mortar of the chosen unit-cells, are considered isotropic elastic and linear. To consider the range of variable elastic characteristics, several numerical simulations are performed in the following section (section 3). The first choice of those material characteristics is reported in Table 2.

Table 2. Initial mechanical elastic properties of blocks and mortar

The deformed unit-cell under $\boldsymbol{E}_{12}$ in-plane shear strain and $\boldsymbol{\chi}_{12}$ out-of-plane torsion strain are presented in Fig. 4 for the running bond pattern and in Fig.5 for the stack and English bond pattern. The colormaps represent the Von Mises stress and the original geometry of the unit-cell is given by a wireframe.

Fig.4. Deformed unit-cell for the running bond masonry under membrane loading (shear) (a) and under torsion loading(b)

Fig.5. Deformed unit-cell for the stack and English bond masonry under membrane loading (shear) (a) and under torsion loading(b) 
The in-plane and out-of-plane homogenized coefficients of the obtained Love-Kirchhoff plates, derived from the three chosen unit-cells corresponding to the different studied arrangements, are tabulated in Table. 3 for the elastic properties of blocks and mortar resumed in Table.2.

Table 3. Homogenized coefficients

To compare these results, the overall representation of the homogenized elastic coefficients is given by the Frobenius norm $\|M\|$ applied to the equivalent tensors as following [20]:

$$
\|M\|=\sqrt{\sum_{i=1}^{3} \sum_{j=1}^{3} M_{i j}{ }^{2}}
$$

Table 4 provides the equivalent elastic moduli corresponding to the wall arrangements under study. The three considered masonry arrangements provide nearly the same membrane and flexural homogenized moduli.

Table 4. Frobenius of the constitute homogenized plates tensors for different bond pattern

This comparison shows a higher stiffness for the running bond pattern wall due to the interlocking of blocks. The texture of the masonry wall plays an important role in the homogenized elastic behavior, inducing the level of the anisotropy at the macroscopic scale, even though the differences are not excessive. In the non-linear range, the influence of the structural arrangement is expected to be much more relevant [16].

\section{Parametric in and out-of-plane homogenized study and data collection}

The studied masonry walls represent composite structures characterized by an overall orthotropic behavior from a phenomenological point of view as shown in the previous section. This orthotropic behavior of masonry arises from the spatial organization of its constituents, their nature, and the complex unit-mortar interaction. Generally, as it was shown [50], the different orthotropic homogenized constants depend on a huge number of parameters such as the geometric arrangement, dimensions and the properties of the constitutive materials of the structure and, the joint thickness. Then, to determine the different homogenized constants, a huge number of masonry typologies should be analyzed. After the validation of the numerical model, presented in the previous part, compared with a 3D full heterogeneous structure not detailed here, the second objective consists in performing a parametric analysis while varying material and geometrical aspects, to understand their influence on the homogenized membrane and flexural constants of the equivalent Love-Kirchhoff plates of stack bond and running bond masonry wall. Thus, a parameterized finite element model is developed. The different factors chosen to be modified in this study are the most influential on the 
response (homogenized coefficients). These various factors are summarized as follows: the ratio between mortar and blocks Yong modulus $\xi=\frac{E^{B}}{E^{M}}$, the thickness of the mortar joint $e^{v}=e^{h}$ (several cases are studied considering infinitely thin and thick joints), the Poisson ratios of both constituent material blocks and mortar $v$, the ratio between the height and the length of blocks $a^{\prime} / b$, and the arrangement of the structure. To be noted, only two commonly used periodic textures masonry walls (stack bond and running bond) are considered in the parametrical analysis due to a large number of numerical simulations needed.

The homogenized $A_{i j k l}$ moduli are normalized versus the corresponding $A_{i j k l}{ }^{B S}$ moduli of the block for the unit-cells constituted by solid concrete blocks without holes, where $A_{i j k l}^{B S}=\frac{E^{B}}{1-v_{b}^{2}} t$. It is noted that the solid blocks have the same material properties compared to those of the hollow one, as well as the same geometrical dimensions. The trend of the normalized homogenized membrane moduli $A_{1111}{ }^{*}=\frac{A_{1111}}{A_{1111}^{B S}}$ of the two masonry wall understudy, varying the ratio $\xi$ for two cases of the thickness of joint mortar (thin and thick joint) is presented in Fig.6 when the Poisson ratios of both constituent material block and mortar ( $v_{b}$ and $v_{m}$ respectively) are assumed identically equal to 0.25 . It can be noted that the sensitivity of the homogenized membrane coefficient $A_{1111}{ }^{*}$ to mortar thickness decreases considerably when $\xi$ increases and the expected difference tends to vanish for $\xi=1$, which is quite obvious because of the limit case representing the homogenous plate. In conclusion, the overall elastic modulus of the material decreases as the mortar joint deformability or thickness increases.

Fig.6. Trend of normalized $A_{1111}{ }^{*}$ membrane modulus as a function of $\mathrm{E}^{\mathrm{B}} / \mathrm{E}^{\mathrm{M}}$ Similarly, homogenized $D_{i j k l}$ moduli are normalized versus the corresponding $D_{i j k l}{ }^{B S}$ moduli of the block for a unit-cell constituted by solid concrete blocks without holes, where the normalized outof-plane factor $D_{i j k l}^{B S}=\frac{E^{B}}{12\left(1-v_{b}^{2}\right)} t^{3}$ corresponds to a homogeneous plate derived from a structure formed by solid concrete blocks. In the same vein, the trend of the normalized homogenized flexural 
coefficient $D_{1111}{ }^{*}=\frac{D_{1111}}{D_{1111}{ }^{B S}}$ is presented in Fig.7 varying $\xi$ for two cases of joint thickness. It can be noted that the sensitivity of the homogenized flexural coefficient to mortar thickness goes down considerably when $\xi$ goes up and the expected difference tends to disappear for $\xi=1$ which is quite obvious because the limit case represents the homogenous plate. Thus, the mortar thickness and the ratio of the Young modulus have a significant effect on the homogenized plate coefficients

Fig.7. Trend of normalized $\mathrm{D}_{1111}$ flexural modulus as a function of $\mathrm{E}^{\mathrm{B}} / \mathrm{E}^{\mathrm{M}}$

The non-homogeneity in the constituents along with the texture (bond pattern) and the thickness of the mortar joints lead to an equivalent structure that is anisotropic in the linear range.

To summarize the performed parametrical analyze indicates that different levels of orthotropy of the elastic equivalent behavior can occur depending on the arrangement, geometry and material properties of the two constituent materials (blocks and mortar). These results provide useful information in cases where the masonry orthotropic description is necessary.

After considering these influent factors, a generalization of the derivation of the homogenized equivalent material is made by ANNs.

\section{Neural Network modeling}

\subsection{Data preparation}

In this section, $\mathrm{ANN}$ is used to overcome the problem of requiring expensive numerical simulation. To train and test the neural network, we need an expanded database test.

For selecting the number of combinations, the procedure of the general full factorial design of experiments is adopted. Therefore, a total number of 192 numerical simulation runs are needed. The domain of variation of different factors is summarized in Table.5. It is noted that the levels of different variables are selected through previous realistic data, and only two bond patterns are studied due to the height number of parametrical numerical simulation required. The material characteristics of both blocks and mortar are taken according to Eurocode 6 [51]. Whereas, the dimensions of blocks are modified according to the NF P14-402 requirements [42].

Table 5. Factors and levels for factorial design

The data are collected based on the factorial design criterion. This data is obtained after several FEM simulations to cover the maximum of material, geometric and arrangement variations. Afterward, 
the obtained data are randomly divided into two groups; 152 data ( $80 \%)$ set are selected as training sets, and the remaining $40(20 \%)$ data were selected as testing sets [52, 53]. The output data are composed of the different homogenized coefficients of the equivalent plates of the hollow concrete block masonry wall.

\subsection{ANN modeling}

ANNs are non-linear statistical data modeling tools inspired from the real human nervous system. One of the characteristics of ANNs is the importance to learn and generalize from examples and experiences to overcome problems where solutions are complicated or where the relationship between input and output data are not evident. ANNs are composed of set interconnected neurons, which receive the data from the input layer and provide a response (output layer) through an inside layer that processes the information. Fig.8 shows the architecture of ANN model. This network is made by a set of neurons acting together. Two steps take place inside each neuron. Firstly, the input values are combined linearly, and each one is multiplied by a corresponding coefficient known as the weight in addition to independent terms or bias. Then the sum of these combinations is applied to a transfer function so as to obtain the output of the neuron. This function can be either linear or non-linear, although generally linear or sigmoid functions are the most commonly used, as shown in Fig.9.

Fig.8. Single neuron activity

The most used ANN is the feedforward, as depicted in Fig.9. In the latter, the neurons are arranged into three groups or layers. The first one represents the input layer, as mentioned above, which contains the network input. The last layer contains the output one. Between the two described layers, we find an intermediate one known as the hidden layer. The number of neurons in each hidden layer depends on the problem to solve [38]. In this presented network, the neurons of each layer are connected with those in the next layer, so the information flows from the input layer to the output one. As precisely explained, a linear combination is executed in each set. Initially, random weight values are used, and then they are corrected through a process known as training. In this process, output values are compared with computed outputs and backpropagate the error see Fig.10. Finally, the values of the adequate weights are obtained by minimizing the ratio between the target output and the measured ones. The most frequently used algorithm with a feedforward multilayers network is the Back-Propagation (BP) algorithm. 
Fig.9. ANN architecture with input, sum function, log-sigmoid activation function, and output

The BP algorithm is the most popular ANN, which is employed for efficient generalization competence. This algorithm represents an iterative gradient one used generally to compute the connection weights minimizing the total mean-square error between the actual output of the multilayer network and the desired output $[54,55]$. Generally, it is the most suitable algorithm for treating nonlinear problems and it is applied to train a feed-forward neural network. The neural- networkbased modeling process involves five main aspects [56]: (1) data acquisition, (2) architecture determination, (3) learning process determination, (4) training of networks and finally (5) validation and testing of the trained one.

The training step is used to activate the network. It consists of two stages: forward and backward stages as presented in Fig.10. In the former stage, the input data pass from the input layer into the hidden one. In the last step, the error between the predicted and the known value is propagated backward from the output layer to the input one. The aim of the "training" function is to learn the relationship between inputs and their corresponding outputs to establish different weights and biases. For the BP algorithm, initially, a random value of biases and weights are used then the networks are compiled with examples provided by getting value for the output that will be different from its real value [38].

Fig.10. Architecture of used back-propagation algorithm

\subsection{Network architecture}

The architecture of the proposed method is summarized in Fig.11. In this research, an in-house ANN program written in FORTRAN is applied. The chosen BP networks have three layers of neurons, as shown in Fig.11.

Fig.11. Flowchart of the proposed ANN

The three layers are summarized in an input layer, a hidden layer, and an output layer. In the first one, no operations are performed on the input data, which are known by the $\mathrm{L}$ neurons. These values 
are directly transmitted to the hidden neurons of the hidden layer affected by weights $w_{i j}$. The single neuron performs a weighted sum of inputs $x_{i}$ that are generally the outputs of the neurons of the previous layer $z_{j}$ adding the threshold value $b_{i}$ and producing an output given by the weighted sum equation (11):

$z_{j}=\sum_{i=1}^{L} w_{i j} x_{i}+b_{i}$

Input signals cumulated in the neuron block are activated by a linear or nonlinear function to have only one input $z_{j}$ given by:

$y_{j}=f\left(z_{j}\right)$

The hidden layer activation function is chosen as the sigmoid one given in equation (13):

$f\left(z_{j}\right)=\frac{1}{1+\exp \left(-\beta z_{i}\right)}$

where $\beta$ represents a parameter defining the slope of the function [57].

\subsection{Preparation of data}

As mentioned above, from the total studied data, $80 \%$ is used to train the networks, and the remaining data are used for testing. The obtained data from FEM are not entered directly in the neural networks but were previously scaled in order to improve the stability of the training process and to obtain a higher degree of accuracy. The scaling of the input to the $[0,1]$ range may improve the training speed, as these normalized values fall in the region of the sigmoid transfer function [58]. The algorithm of the data normalization is given by Eq.14:

$x_{n}=\frac{y_{\text {max }}-y_{\text {min }}}{x_{\text {max }}-x_{\text {min }}}\left(x-x_{\text {min }}\right)+y_{\text {min }}$

where $x_{n}$ is the normalized value of the variable $x, x_{\max }$ and $x_{\min }$ are respectively the maximum and minimum value of $x, y_{\max }$ and $y_{\min }$ are respectively the maximum and minimum value of the normalized target. Since the chosen normalized target is scaled in the $[0,1]$ range, the normalization function can be expressed as:

$x_{n}=\frac{x-x_{\min }}{x_{\max }-x_{\min }}$ 


\subsection{Training algorithm}

The training process in ANNs involves presenting a set of examples acquired from experiments or numerical calculation (input patterns) with known outputs (target output). As mentioned previously, the chosen networks for this paper is BP. The learning used algorithm can be summarized as follows [57]: the first step consists in selecting the learning rate defined by $\eta=0.01$ and the momentum coefficient $\alpha=0.01$. After that, a group of random numbers within $(-1,1)$ is taken as the initial value of the weight. Next, the outputs of all neurons layer by layer are computed starting with the input layer and using Eq. (10) and (11). The fourth step consists of computing the mean square error MSE between the value of the output of the network and that desired according to the next relationship:

$$
M S E=\frac{1}{2} \frac{1}{P} \sum_{j=1}^{P} \sum_{i=1}^{N}\left(D_{i j}-y_{i j}\right)^{2}
$$

where $N$ is the number of output nodes, $P$ is the total number of patterns, $D_{i j}$ represents the desired outputs (numerical values) and $y_{i j}$ is the predicted output of networks. Afterward, if the MSE is small enough, then stop learning. Then the learning errors for every neuron is computed layer by layer, as given in Eq.17.

$\delta_{j}=\left(D_{j}-y_{j}\right) z_{j}$

Step 7 is dedicated to updating the weight along the negative gradient of error and finally, repeat the third step. It is that noted the number of epochs affects the rentability of networks, where the epoch represents the entire set of training patterns to the network.

In order to guarantee the efficiency of the considered neural networks, a series of trails should be made varying some intrinsic parameters that minimize the MSE of the training data. For the performance evaluation of the trained model, other indicators can be used, such as the root mean square error (RMSE) expressed by Eq.18, the coefficient of determination $\left(R^{2}\right)$ expressed by Eq.19 and the mean absolute percentage error (MAPE) expressed by Eq.20:

RMSE statistics compares the measured values to the predicted one and computes the square root of the average residual error. It falls when performance rises. A lower RMSE value indicates a good prediction performance of the model. The prediction performance is considered perfect when the latter is equal to 0 , the RMSE gives more weightage to large errors [58].

$$
R M S E=\sqrt{\frac{1}{2} \frac{1}{P} \sum_{j=1}^{P} \sum_{i=1}^{N}\left(D_{i j}-y_{i j}\right)^{2}}
$$


The coefficient of determination computes the strength of similarity between the two considered variables. It depends on the linear relationship between the measured values and the predicted ones. A value close to unity indicates the accuracy of the model.

$$
R^{2}=1-\frac{\sum_{i=1}^{n}\left(D_{i}-y_{i}\right)^{2}}{\sum_{i=1}^{n}\left(D_{i}-y_{\text {avg }}\right)^{2}}
$$

where $y_{\text {avg }}$ represents the average of measured values.

The MAPE is a nondimensional statistics coefficient that provides an effective way of comparing the residual error for each data point with respect to the target value. Smaller MAPE values indicate a better performance of the model and vice versa.

$$
\text { MAPE }=\frac{1}{N} \sum_{i=1}^{N}\left|\frac{D_{i}-y_{i}}{y_{i}}\right| \times 100
$$

\subsection{Network selection}

It can be stated that the prediction performance of a network largely depends on its parameters and chosen architecture [59]. Obtaining the best network for a particular case is a difficult task that generally requires successive trials as well as extensive parametric studies. Network architecture consists of different numbers of hidden layers, and processing elements are created and trained with various network parameters. The performance is mainly associated with the minimum square error in the tested set [53]. In this way, a lot of networks that are capable of generalization at different levels are obtained, and the best network is selected from these many network candidates. In the current study, the network performance in terms of RMSE is measured and plotted after every iteration, and the lowest training error is at 10,000 iterations (epochs), as shown in Fig.12. It can be demonstrated that after this number of training cycles, the networks curve is in good agreement with the FEM ones. As a conclusion, this ANN has been sufficiently trained where the maximum error is less than $1.5 \%$.

Fig.12. RMSE for training datasets versus the number of iterations for ANN model

For statistical modeling, only one hidden layer is often satisfactory [60]. One of the key issues in designing a multilayer network is to find the optimum number of neurons in the hidden layer. Therefore, the performance of the model with a system of one hidden layer is studied, by varying 
the number of neurons. Unfortunately, there is no direct method available to estimate the number of hidden layers and the number of neurons in each hidden layer. A network having too many numbers of neurons may cause overfitting. The optimal number of neurons in the hidden layers is also predicted by the trial and error method based on the minimum RMSE [61]. Fig.13 presents the RMSE trend versus a variable number of neurons in the hidden layer. The lowest training error is obtained for 14 neurons in the hidden layer.

Fig.13. Influence of the number of hidden neurons on RMSE

After a series of trails, the values of the parameters that minimize the MSE of the training data are selected as follows:

Number of input layer units: 5

Number of hidden layers: 1

Number of hidden layer units: 14

Number of output layer units: 8

Activation function: sigmoid function

Momentum rate: 0.1

Learning rate: 0.01

Error after learning: 0.0129

Learning cycle: 10,000

\subsection{Network results and discussion}

Once the ANN model satisfies the desired error, the performance of the selected ANN network models for training and testing can be seen in Fig. 14 and Fig. 15, respectively. It is worth noting that the process responses of interest presented in this study are only two equivalent coefficients: the normalized membrane elastic constant $A_{1111}{ }^{*}$ and the normalized flexural elastic constant $D_{1111}{ }^{*}$ of the homogenized equivalent plate. For both figures: (a) represents the curves for the normalized membrane elastic constant and (b) represents the curves for the normalized flexural elastic constant. In Fig. 14 and Fig. 15, a superposition of the results of the ANN model output and the actual finite element data for the equivalent elastic constant is presented. The horizontal axis stands for the sample numbers, and the vertical one is their corresponding normalized elastic responses. For both chosen constants, it can be clearly seen that the predicted values of the training and testing sets in the constructed ANN model are very close to the target values, indicating that the ANN model can successfully learn the nonlinear relationship between the input and output variables with a high level 
of accuracy. More precisely, for the training result, the maximum error between the ANN results compared to that calculated by FEM is 0.058 for the normalized membrane elastic constant and is 0.067 for the normalized flexural elastic constant as shown Fig.14.

Fig.14. Evaluation of numerical and predicted coefficient by ANN for training phase: (a) Normalized membrane elastic constant; (b) Normalized flexural elastic constant

For the testing data, the maximum error between the ANN results compared to that calculated by FEM remains less than 0.12 for all homogenized constants. Therefore, the selected model shows good potential for predicting the homogenized constant of equivalent masonry structures.

Fig.15. Evaluation of numerical and predicted coefficient by ANN for testing phase: (a) Normalized membrane elastic constant; (b) Normalized flexural elastic constant

Another way to present this comparison between the two models is illustrated in Fig. 16 and Fig. 17. Likewise, for both figures, (a) represents the curves for the normalized membrane elastic constant and (b) represents the curves for the normalized flexural elastic constant. These figures present the linear regression between the predicted results of the best-selected network model. The theoretical 1:1 line is also drawn to show the overall trend. We can see that all the points are scattered around this line. This could be explained by the robustness and effectiveness of the neural algorithm to the prediction of results very close to those calculated by FEM. From Fig. 15 and Fig. 17, it can be noted that the constructed ANN model is well learned and is able to generate other responses for other input parameters different from those applied in the learning phase. The performance indicator is evaluated using the coefficient of determination $\left(R^{2}\right)$. The results show a better fit in the training set than in the testing set. It can be seen that the coefficient of determination value $R^{2}$ is 0.998 for the training set and 0.977 for the testing set.

Fig.16. Comparison of ANN output with numerical values for training phase: (a) Normalized $A_{1111}$ (b) Normalized $D_{1111}$

In terms of cost and calculation time, it can be noted that computation by the developed algorithm is faster than the FEM simulation for predicting such a response. The time required to carry out the FEM calculation lasts 50 minutes (for only one test) while by applying the ANN model the 
prediction time of the responses is of the order of a few seconds. This demonstrates that the proposed algorithm is a good estimator, which is able to predict the results obtained by FEM in a very short time.

Fig.17. Comparison of ANN output with numerical values for testing phase: (a) Normalized $A_{1111}$;

(b) Normalized $D_{1111}$

The results of these figures indicate that the ANN model can successfully learn and establish the relationship between different input parameters and desired outputs. Table 6 provides the results of the model of the back-propagation network measured by $R^{2}, R M S E$, and MAPE for all the equivalent plate coefficients. It can be seen that the coefficient of determination $R^{2}$ value in the training set varies from 0.9956 to 0.9982 . On the other hand, it barely gets down for the testing phase varying from 0.94 to 0.982 . The highest values of $M A P E$ and $R M S E$, in the testing phase, are $5.41 \%$ and $5.8 \%$, respectively. Therefore, the constructed ANN model is able to provide the prediction of the homogenized plate coefficients with high precision.

Table 6. Coefficients of determination of normalized responses

\section{Conclusions}

In this paper, a new and efficient intelligent approach is proposed to estimate the different homogenized coefficients of the equivalent Love-Kirchhoff plates of different bond pattern masonry walls formed by hollow concrete blocks bonded by mortar. The database used to construct the ANNs is collected from a numerical periodic homogenization in several parameters. Firstly, the homogenized elastic properties are numerically derived. The membrane and bending elastic moduli of the equivalent Love-Kirchhoff plates are determined by the homogenization scheme on a periodic adequately chosen unit-cells. A specific Python program using Abaqus software is developed to automatically program the applied periodic boundary conditions and the post-processing stages. Since the studied walls are characterized by an overall orthotropic behavior, the different derived orthotropic homogenized constants are a function of a huge number of parameters such as the geometric arrangements, dimensions and the material properties of both blocks and mortar. Then, a statistical analysis is conducted to investigate the number of finite-element simulation and to ensure all the combinations between different studied parameters. 
Based on the previously collected database, $A N N$ is trained and tested to predict the equivalent behavior of the studied structures. The network was trained by the back-propagation algorithm. Network architecture with a different number of nodes in hidden layers and of iterations has been examined. It has been indicated that the learning and prediction performances of the networks depend on the number of neurons in the hidden layer as well as the appropriate network parameters. The minimum square error was obtained for fourteen neurons in the hidden layer. Once the network is trained properly, the results showed a good agreement with respect to the finite element data. This efficiency can be proven in terms of higher coefficient of determination and lower RSME and MAPE. In general, the proposed $A N N$ can predict the effective properties of the equivalent Love-Kirchhoff plate of different bond pattern masonry walls with satisfactory accuracy, reliability, and low computational effort.

Future work may be extended to predict the surface failure of different masonry structures constituted by hollow blocks using ANNs.

\section{Acknowledgments}

The authors gratefully acknowledge the researchers Abdessalam CHAMEK and Hédi BEL HADJ SALAH for supplying the Fortran code for the neural network analysis without which this work would not have been possible.

\section{Conflict of Interest:}

The authors declare that they have no conflict of interest. 


\section{References}

1. Nino S Di, Luongo A (2019) A simple homogenized orthotropic model for in-plane analysis of regular masonry walls. Int J Solids Struct

2. Drougkas A, Verstrynge E, Hayen R, Van Balen K (2019) The confinement of mortar in masonry under compression: Experimental data and micro-mechanical analysis. Int J Solids Struct 162:105-120

3. Pantò B, Silva L, Vasconcelos G, Lourenço PB (2019) Macro-modelling approach for assessment of out-of-plane behavior of brick masonry infill walls. Eng Struct 181:529-549

4. Salerno G, de Felice G (2009) Continuum modeling of periodic brickwork. Int J Solids Struct 46:1251-1267

5. Petracca M, Pelà L, Rossi R, et al (2017) Micro-scale continuous and discrete numerical models for nonlinear analysis of masonry shear walls. Constr Build Mater 149:296-314

6. Cecchi A, Sab K (2002) A multi-parameter homogenization study for modeling elastic masonry. Eur J Mech A/Solids 21:249-268

7. Anthoine A (1995) Derivation of the in-plane elastic characteristics of masonry through homogenization theory. Int J Solids Struct 32:137-163

8. Stefanou I, Sab K, Heck J-V (2015) Three dimensional homogenization of masonry structures with building blocks of finite strength: A closed form strength domain. Int J Solids Struct 54:258-270

9. Cavalagli N, Cluni F, Gusella V (2011) Strength domain of non-periodic masonry by homogenization in generalized plane state. Eur J Mech A/Solids 30:113-126

10. Friaa H, Hellara M, Stefanou I, et al (2018) In-plane strength domain numerical determination of hollow concrete block masonry. In: Design and Modeling of Mechanical Systems-III. CMSM 2017. Lecture Notes in Mechanical Engineering. Springer

11. Cecchi A, Sab K (2004) A comparison between a 3D discrete model and two homogenised plate models for periodic elastic brickwork. Int J Solids Struct 41:2259-2276

12. Bacigalupo A, Gambarotta L (2012) Computational two-scale homogenization of periodic masonry: Characteristic lengths and dispersive waves. Comput Methods Appl Mech Eng 213-216:16-28

13. Mistler M, Anthoine A, Butenweg C (2007) In-plane and out-of-plane homogenisation of masonry. Comput Struct 85:1321-1330

14. Caporale A, Parisi F, Asprone D, et al (2014) Micromechanical analysis of adobe masonry as two-component composite : Influence of bond and loading schemes. Compos Struct 112:254-263

15. Silva LC, Lourenço PB, Milani G (2018) Derivation of the out-of-plane behaviour of masonry through homogenization strategies: Micro-scale level. Comput Struct 209:30-43

16. Milani G, Cecchi A (2013) Compatible model for herringbone bond masonry: Linear elastic homogenization, failure surfaces and structural implementation. Int J Solids Struct 50:32743296

17. Cluni F, Gusella V (2004) Homogenization of non-periodic masonry structures. Int J Solids Struct 41:1911-1923

18. Cecchi A, Sab K (2009) Discrete and continuous models for in plane loaded random elastic brickwork. Eur J Mech A/Solids 28:610-625

19. Gusella V, Cluni F (2006) Random field and homogenization for masonry with non periodic microstructure. J Mech Mater Struct 1:97-127

20. Cavalagli N, Cluni F, Gusella V (2013) Evaluation of a Statistically Equivalent Periodic Unit Cell for a quasi-periodic masonry. Int J Solids Struct 50:4226-4240

21. Sab K (2009) Overall ultimate yield strength of a quasi-periodic masonry. Comptes Rendus - Mec 337:603-609

22. Milani G, Esquivel YW, Lourenço PB, et al (2013) Characterization of the response of 
quasi-periodic masonry: Geometrical investigation, homogenization and application to the Guimarães castle, Portugal. Eng Struct 56:621-641

23. Cavalagli N, Cluni F, Gusella V (2018) Failure surface of quasi-periodic masonry by means of Statistically Equivalent Periodic Unit Cell approach. Meccanica 53:1719-1736

24. Cecchi A, Di Marco R (2000) Homogenization of masonry walls with a computational oriented procedure. Rigid or elastic block? Eur J Mech A/Solids 19:535-546

25. Drougkas A, Roca P, Molins C (2015) Analytical micro-modeling of masonry periodic unit cells - Elastic properties. Int J Solids Struct 69-70:169-188

26. Adeli H (2001) Neural Networks in Civil Engineering : 1989 - 2000. Comput Civ Infrastruct Eng 16:126-142

27. Moosazadeh S, Namazi E, Aghababaei H, et al (2018) Prediction of building damage induced by tunnelling through an optimized artificial neural network. Eng Comput 1-13

28. Asteris PG, Plevris V (2016) Anisotropic masonry failure criterion using artificial neural networks. Neural Comput Appl

29. Tayfur G, Erdem TK, Kira Ö (2014) Strength Prediction of High-Strength Concrete by Fuzzy Logic and Artificial Neural Networks. J Mater Civ Eng 10:1-7

30. Duan ZH, Kou SC, Poon CS (2013) Using artificial neural networks for predicting the elastic modulus of recycled aggregate concrete. Constr Build Mater 44:524-532

31. Eskandari-Naddaf H, Kazemi R (2017) ANN prediction of cement mortar compressive strength , influence of cement strength class. Constr Build Mater 138:1-11

32. Hammoudi A, Moussaceb K, Belebchouche C, Dahmoune F (2019) Comparison of artificial neural network (ANN) and response surface methodology (RSM) prediction in compressive strength of recycled concrete aggregates. Constr Build Mater 209:425-436

33. Plevris V, Asteris PG (2014) Modeling of masonry failure surface under biaxial compressive stress using Neural Networks. Constr Build Mater 55:447-461

34. Zhou Q, Zhu F, Yang X, et al (2017) Shear capacity estimation of fully grouted reinforced concrete masonry walls using neural network and adaptive neuro-fuzzy inference system models. Constr Build Mater 153:937-947

35. Zhang Y, Zhou GC, Xiong Y, Rafiq MY (2010) Techniques for Predicting Cracking Pattern of Masonry Wallet Using Artificial Neural Networks and Cellular Automata. J Comput Civ Eng 24:161-172

36. Ghaleini EN, Koopialipoor M, Momenzadeh M, et al (2018) A combination of artificial bee colony and neural network for approximating the safety factor of retaining walls. Eng Comput 1-12

37. Garzón-Roca J, Adam JM, Sandoval C, Roca P (2013) Estimation of the axial behaviour of masonry walls based on Artificial Neural Networks. Comput Struct 125:145-152

38. Garzón-Roca J, Obrer CM, Adam JM (2013) Compressive strength of masonry made of clay bricks and cement mortar : Estimation based on Neural Networks and Fuzzy Logic. 48:21-27

39. Nguyen H, Moayedi H, Foong LK, et al (2019) Optimizing ANN models with PSO for predicting short building seismic response. Eng Comput 35:

40. Zhou G, Pan D, Xu X, Rafiq YM (2009) Innovative ANN Technique for Predicting Failure/Cracking Load of Masonry Wall Panel under Lateral Load. J Comput Civ Eng 24:377-387

41. Barbieri A, Cecchi A (2007) Analysis of masonry columns by a 3D F.E.M homogenisation procedure. In: Proceedings of the 2nd IASME / WSEAS International Conference on Continuum Mechanics (CM’07), Portoroz, Slovenia, May 15-17, 2007. Venice ITALY, pp $68-75$

42. NF P 14-402 (1983) Blocs en béton pour murs et cloisons - Dimensions et tolérances. AFNOR 301:1-15 
43. Caillerie D, Nedelec JC (1984) Thin elastic and periodic plates. Math Meth Appl Sci 6:159191

44. Cecchi A, Milani G, Tralli A (2005) Validation of analytical multiparameter homogenization models for out-of-plane loaded masonry walls by means of the finite element method. J Eng Mech 131:185-198

45. Sab K, Lebée A (2015) Homogenization of Heterogeneous Thin and Thick Plates. Wiley, New York

46. Nemat-Nasser S, Iwakuma T, Hejazi M (1982) On composites with periodic structure. Mech Mater 1:239-267

47. Steven GP (1997) Homogenization of multicomponent composite orthotropic materials using FEA. Numer Methods Eng 13:517-531

48. Lebée A, Sab K (2011) A bending-gradient model for thick plates, Part II : Closed-form solutions for cylinfrical bending of laminates. Int J Solids Struct 48:2889-2901

49. Omairey SL, Dunning PD, Sriramula S (2018) Development of an ABAQUS plugin tool for periodic RVE homogenisation. Eng Comput 35:1-11

50. Lee JS, Pande GN, Kralj B (1998) A comparative study on the approximate analysis of masonry structures. Mater Struct Constr 31:473-479

51. British Standard (BS EN 1996-1-1). Eurocode 6: Design of masonry structures. British Standard Institution. London

52. Kamble L V., Pangavhane DR, Singh TP (2015) Neural network optimization by comparing the performances of the training functions -Prediction of heat transfer from horizontal tube immersed in gas-solid fluidized bed. Int J Heat Mass Transf 83:337-344

53. Demuth H, Mark B (2002) Neural Network Toolbox user's guide, Version 4. The Mathworks Inc, Natick, MA

54. Golafshani EM, Rahai A, Sebt MH, Akbarpour H (2012) Prediction of bond strength of spliced steel bars in concrete using artificial neural network and fuzzy logic. Constr Build Mater 36:411-418

55. Saridemir M (2009) Predicting the compressive strength of mortars containing metakaolin by artificial neural networks and fuzzy logic. Adv Eng Softw 40:920-927

56. Duan ZH, Kou SC, Poon CS (2013) Prediction of compressive strength of recycled aggregate concrete using artificial neural networks. Constr Build Mater 40:1200-1206

57. Khaterchi H, Chamekh A, Belhadjsalah H (2015) Artificial Neural Network Analysis for Modeling Fibril Structure in Bone. 16:581-587

58. Chandwani V, Agrawal V, Nagar R (2015) Modeling slump of ready mix concrete using genetic algorithms assisted training of Artificial Neural Networks. Expert Syst Appl 42:885-893

59. Cachim PB (2011) Using artificial neural networks for calculation of temperatures in timber under fire loading. Constr Build Mater 25:4175-4180

60. Wilamowski BM, Chen Y, Aleksander M (1999) Efficient Algorithm for Training Neural Networks with one Hidden Layer. Proc Int Jt Conf Neural Networks (IJCNN'99) IEEE $1725-1728$

61. Gholamnejad J, Bahaaddini HR, Rastegar M (2013) Prediction of the deformation modulus of rock masses using Artificial Neural Networks and Regression methods. J Min Environ $4: 35-43$ 


\section{Figure captions}

Fig.1. Periodic masonry walls understudy

Fig.2. Chosen unit-cells for masonry walls (a) running bond wall (b) stack bond wall (c) English bond wall

Fig.3. Applied boundary conditions on one-eighth unit-cell (a) for symmetric loading, (b) for skew-symmetric loading

Fig.4. Deformed unit-cell for the running bond masonry under membrane loading (shear) (a) and under torsion loading(b)

Fig.5. Deformed unit-cell for the stack bond and English bond masonry under membrane loading (shear) (a) and under torsion loading (b)

Fig.6. Trend of normalized $A_{1111}{ }^{*}$ membrane modulus as a function of $\mathrm{E}^{\mathrm{B}} / \mathrm{E}^{\mathrm{M}}$

Fig.7. Trend of normalized $D_{1111}{ }^{*}$ flexural modulus as a function of $\mathrm{E}^{\mathrm{B}} / \mathrm{E}^{\mathrm{M}}$

Fig.8. Single neuron activity

Fig.9. ANN architecture with input, sum function, log-sigmoid activation function, and output

Fig.10. Architecture of used back-propagation algorithm

Fig.11. Flowchart of the proposed ANN

Fig.12. RMSE for training datasets versus the number of iterations for ANN model

Fig.13. Influence of the number of hidden neurons on RMSE

Fig.14. Evaluation of numerical and predicted coefficient by ANN for training phase: (a)

Normalized membrane elastic constant; (b) Normalized flexural elastic constant

Fig.15. Evaluation of numerical and predicted coefficient by ANN for testing phase: (a) Normalized membrane elastic constant; (b) Normalized flexural elastic constant

Fig.16. Comparison of ANN output with numerical values for training phase: (a) Normalized $A_{1111}$; (b) Normalized $D_{1111}$

Fig.17. Comparison of ANN output with numerical values for testing phase: (a) Normalized $A_{1111}$; (b) Normalized $D_{1111}$ 


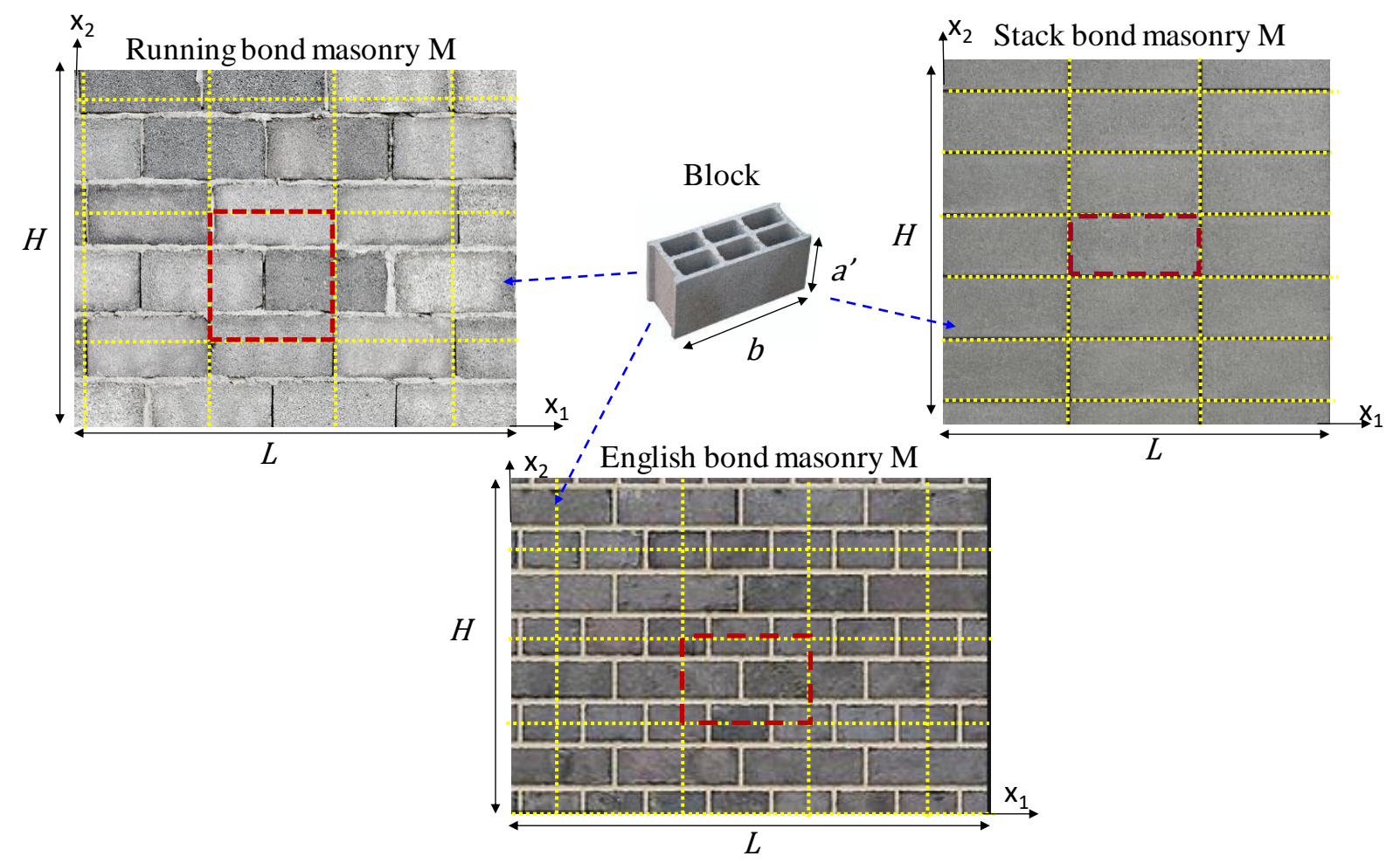

Fig.1. Periodic masonry walls understudy

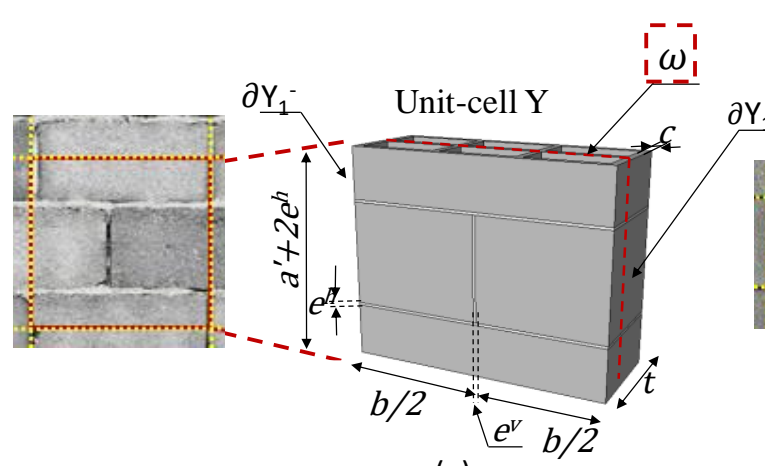

(a)

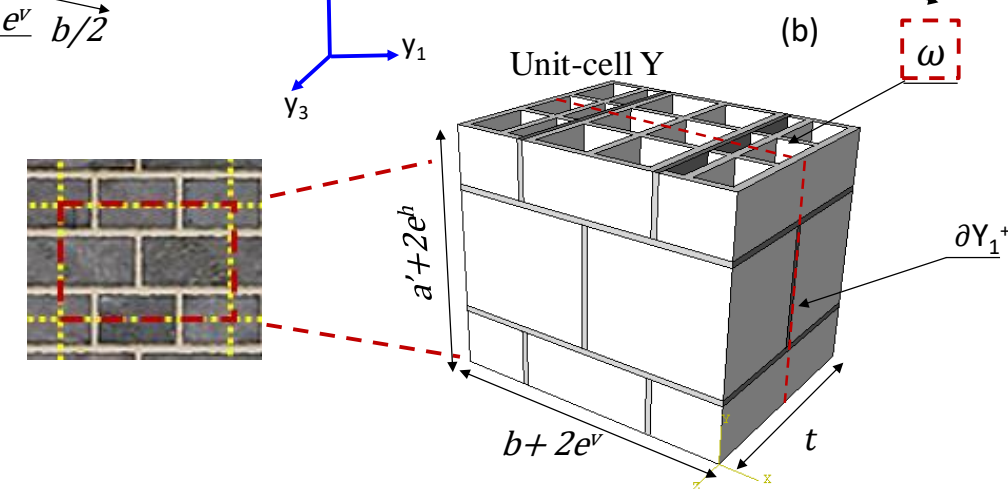

(c)

Fig.2. Chosen unit-cells for masonry walls (a) running bond wall (b) stack bond wall (c) English bond wall 


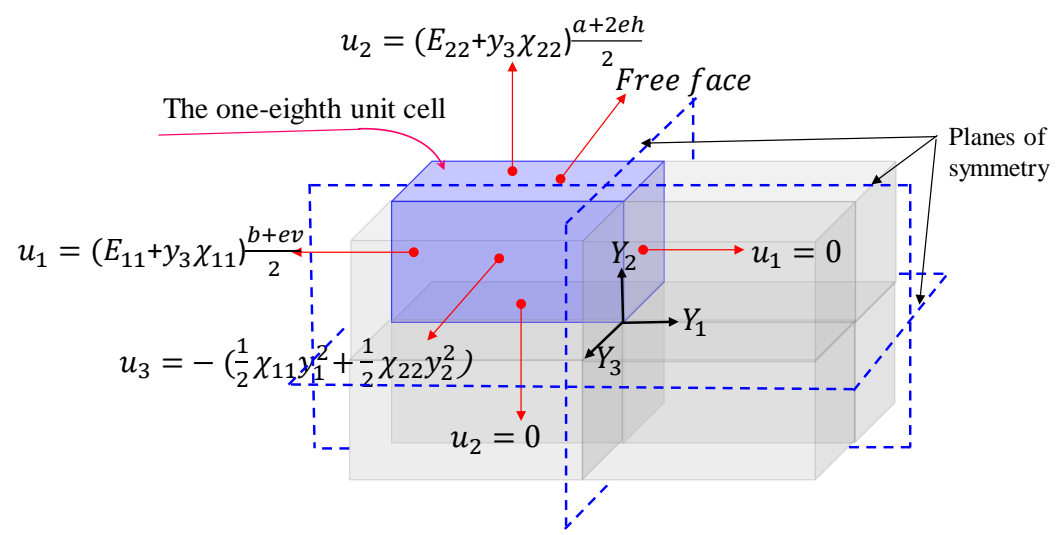

(a) Symmetric loading

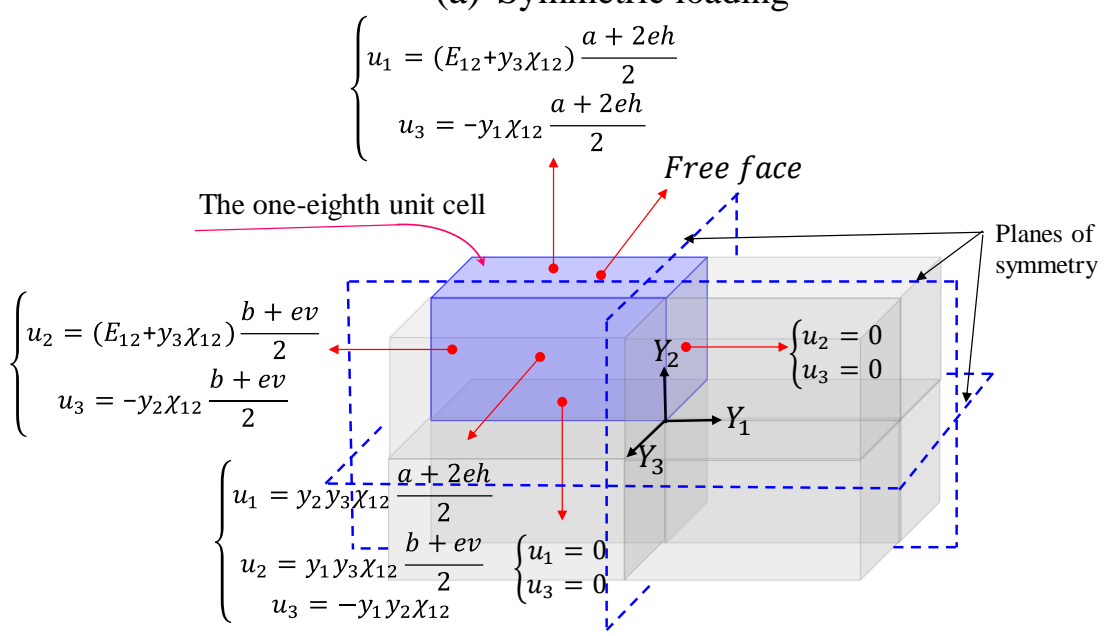

(b) skew-symmetric loading

Fig.3. Applied boundary conditions on one-eighth unit-cell (a) for symmetric loading, (b) for skew-symmetric loading

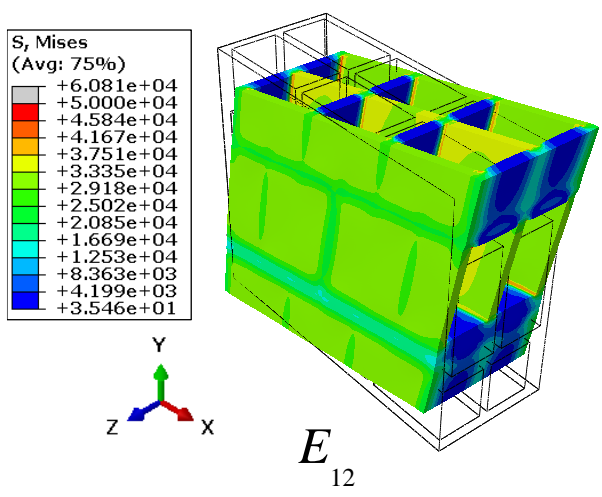

(a)

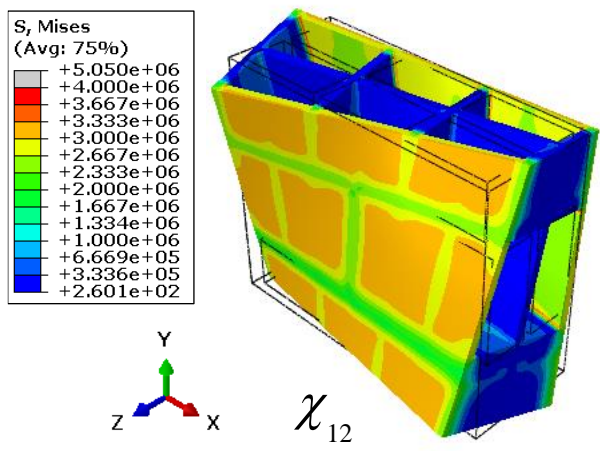

(b)

Fig.4. Deformed unit-cell for the running bond masonry under membrane loading (shear) (a) and under torsion loading(b) 


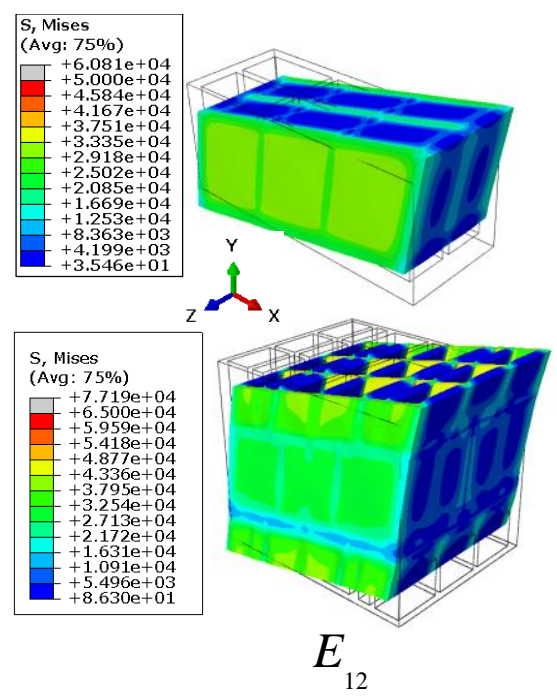

(a)

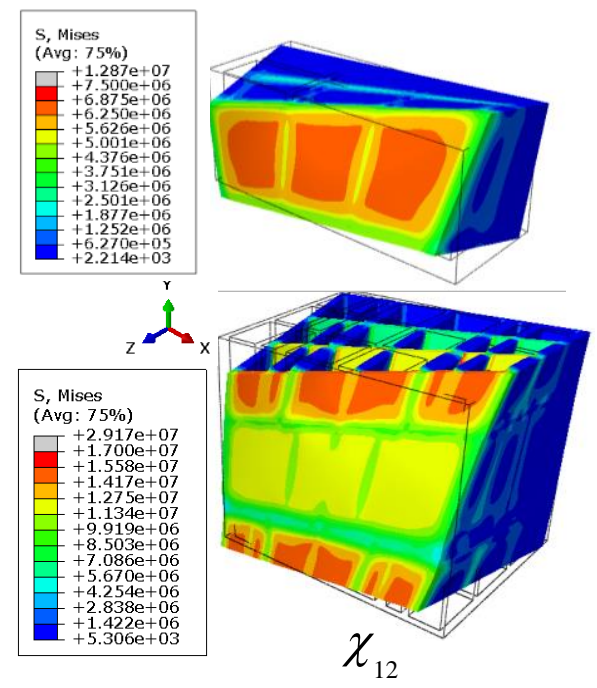

(b)

Fig.5. Deformed unit-cells for the stack bond and English bond masonry under membrane loading (shear) (a) and under torsion loading(b)

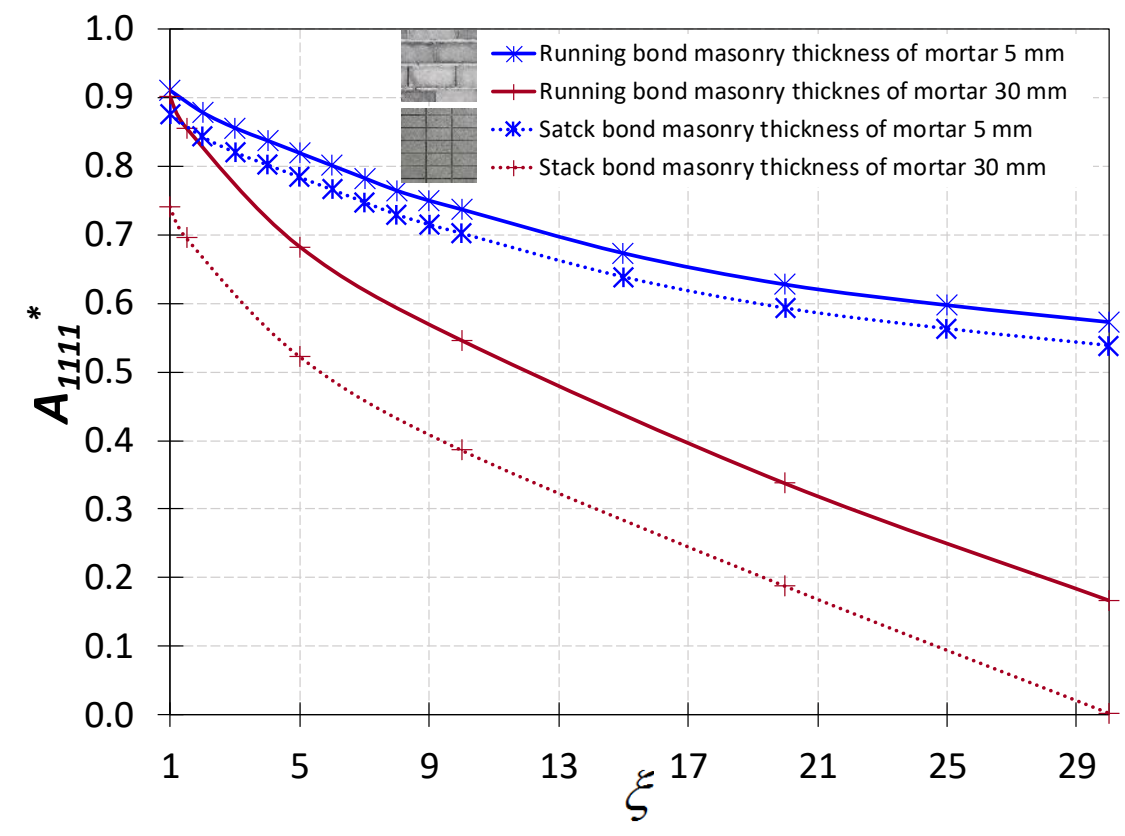

Fig.6. Trend of normalized $A_{1111}{ }^{*}$ membrane modulus as a function of $\mathrm{E}^{\mathrm{B}} / \mathrm{E}^{\mathrm{M}}$ 


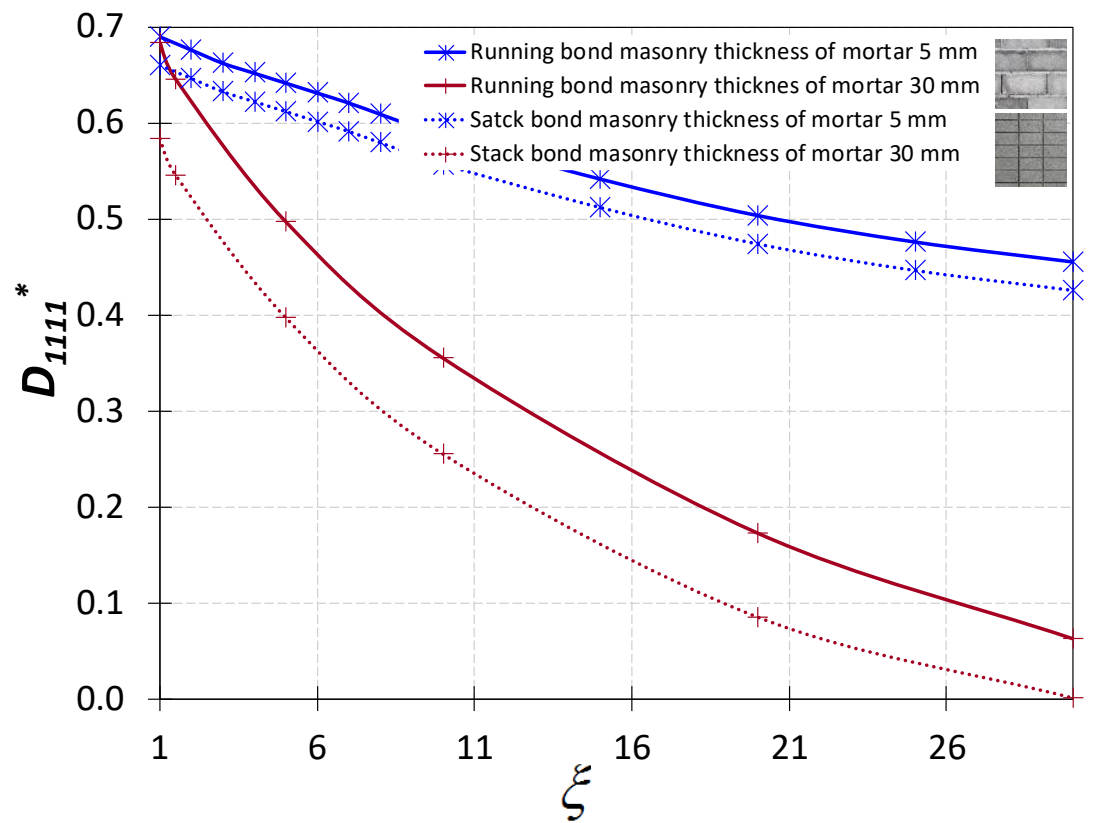

Fig.7. Trend of normalized $D_{1111}{ }^{*}$ flexural modulus as a function of $\mathrm{E}^{\mathrm{B}} / \mathrm{E}^{\mathrm{M}}$

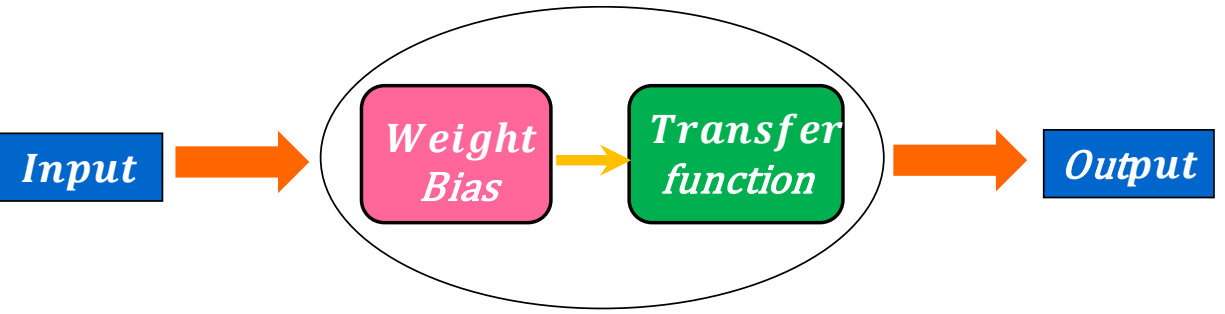

\section{Neuron}

Fig.8. Single neuron activity 


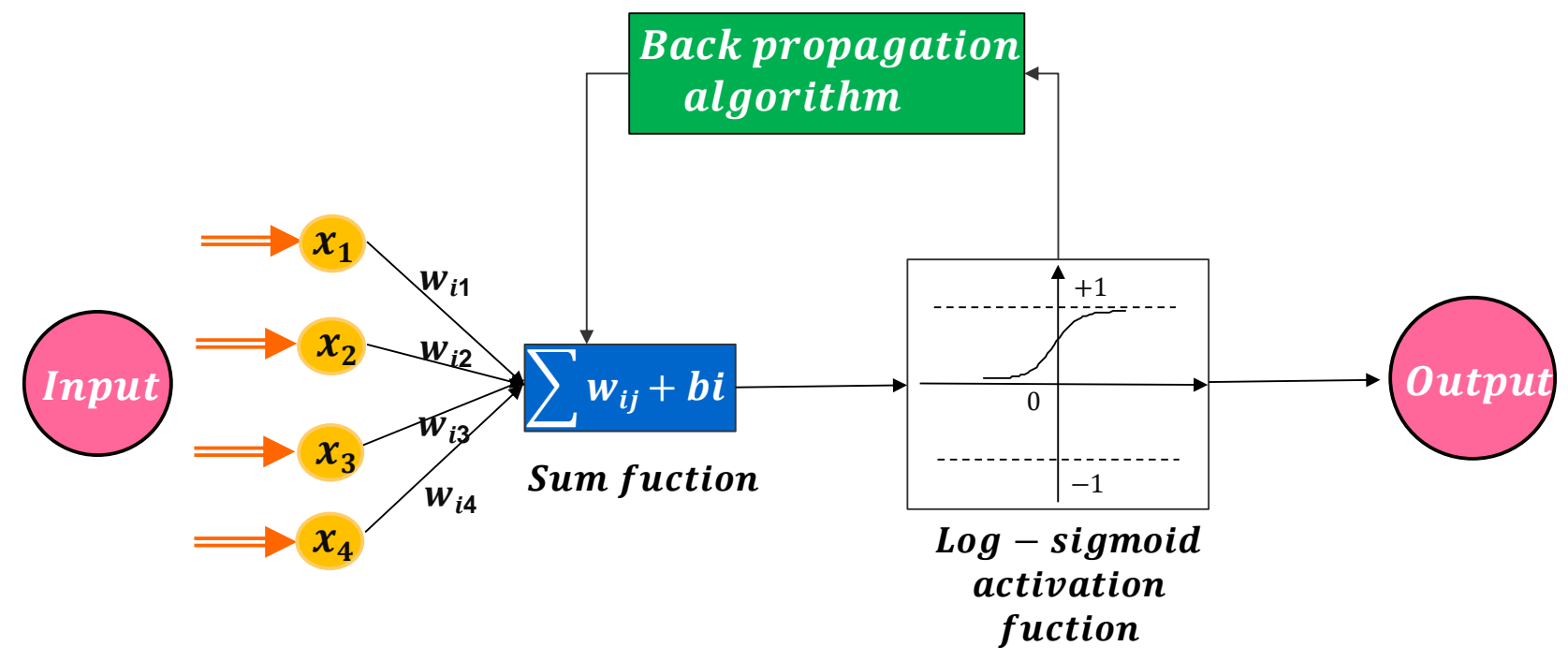

Fig.9. ANN architecture with input, sum function, log-sigmoid activation function, and output

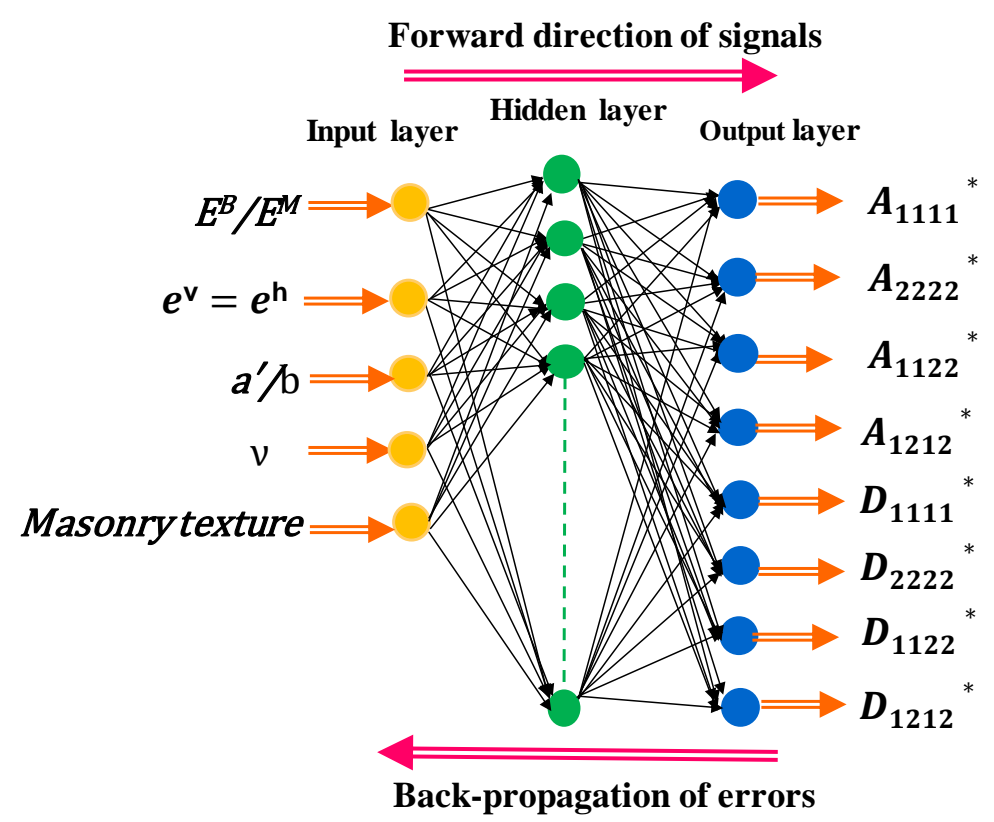

Fig.10. Architecture of used back-propagation algorithm 


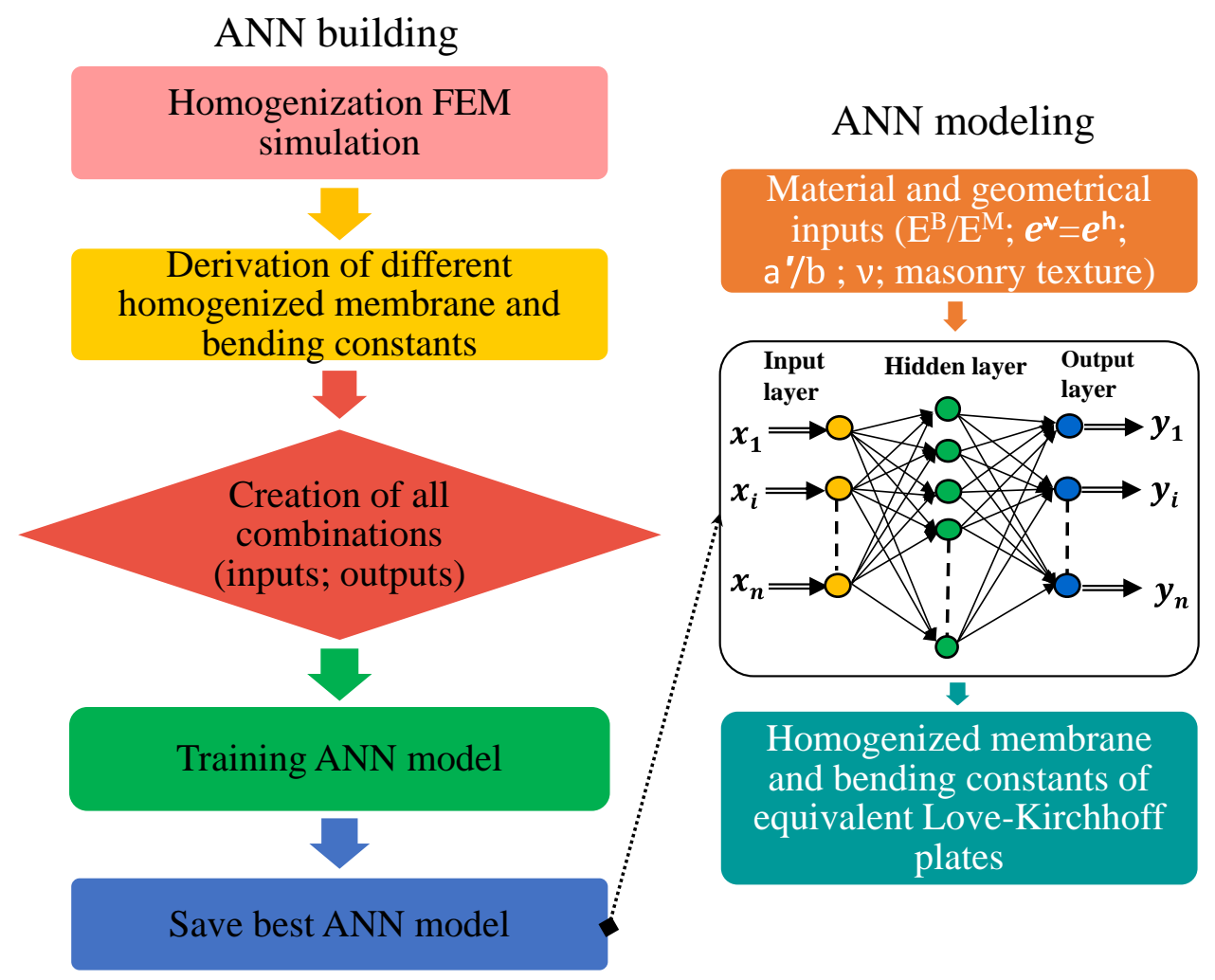

Fig.11. Flowchart of the proposed ANN

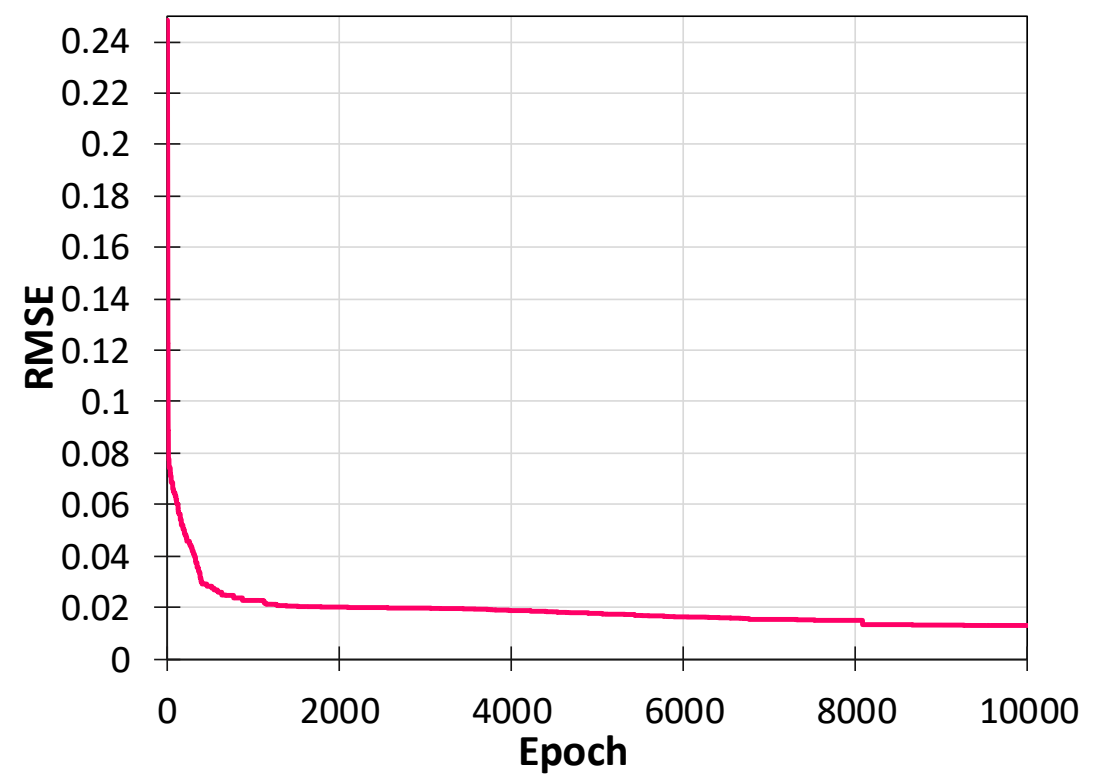

Fig.12. RMSE for training datasets versus the number of iterations for ANN model 


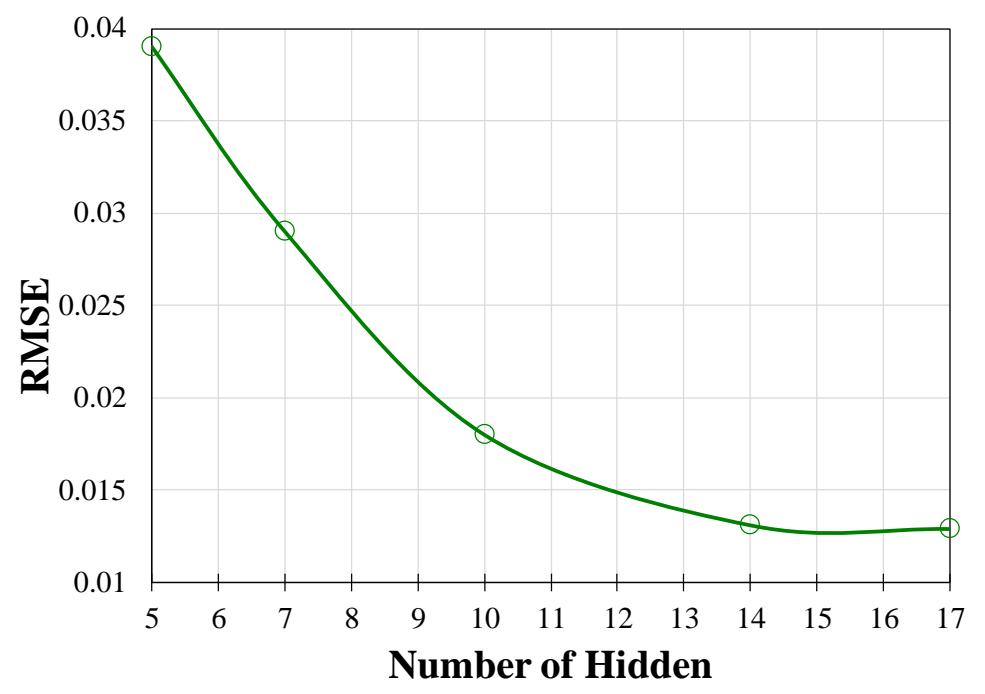

Fig.13. Influence of the number of hidden neurons on RMSE 


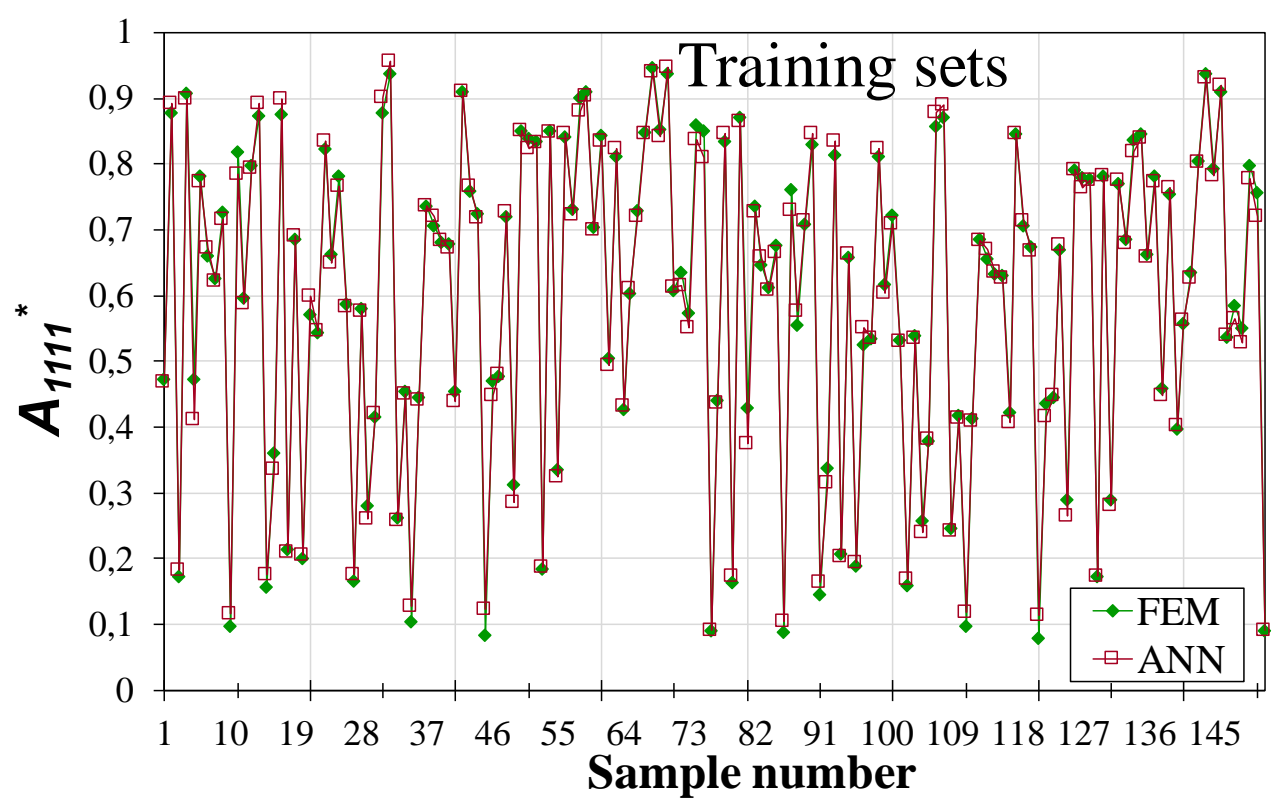

(a)

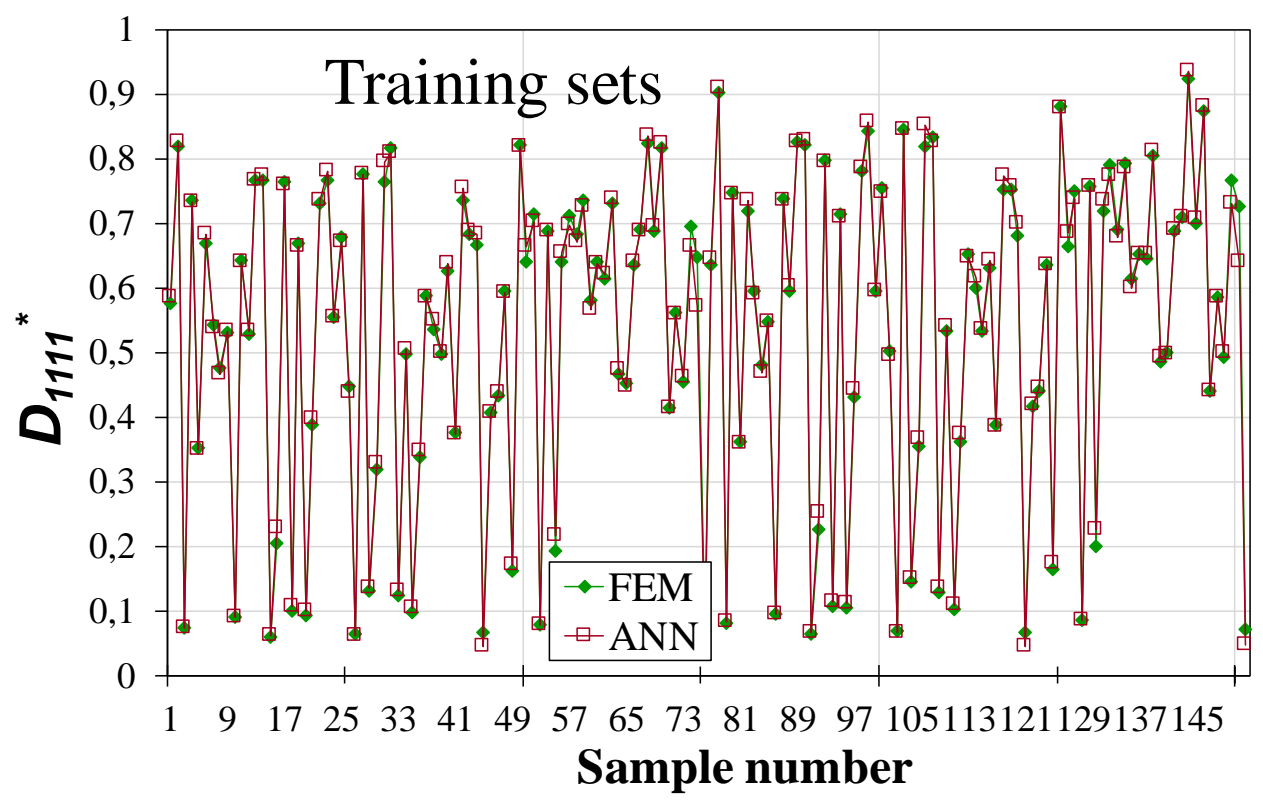

(b)

Fig.14. Evaluation of numerical and predicted coefficient by ANN for training phase: (a) Normalized membrane elastic constant; (b) Normalized flexural elastic constant 


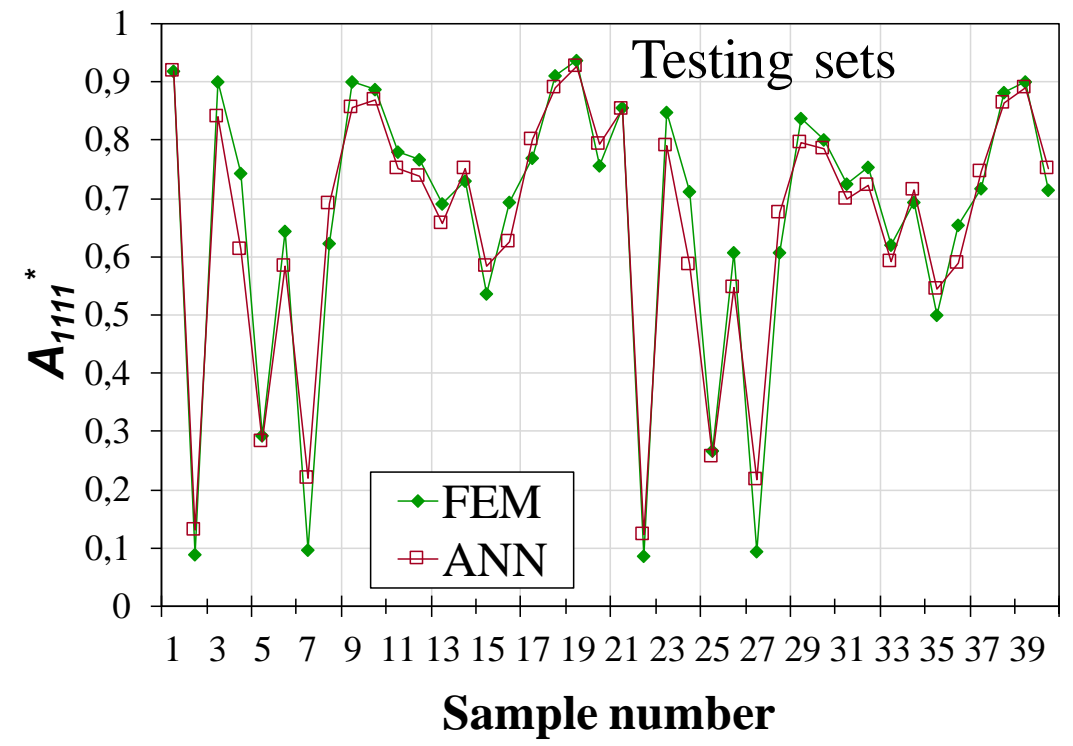

(a)

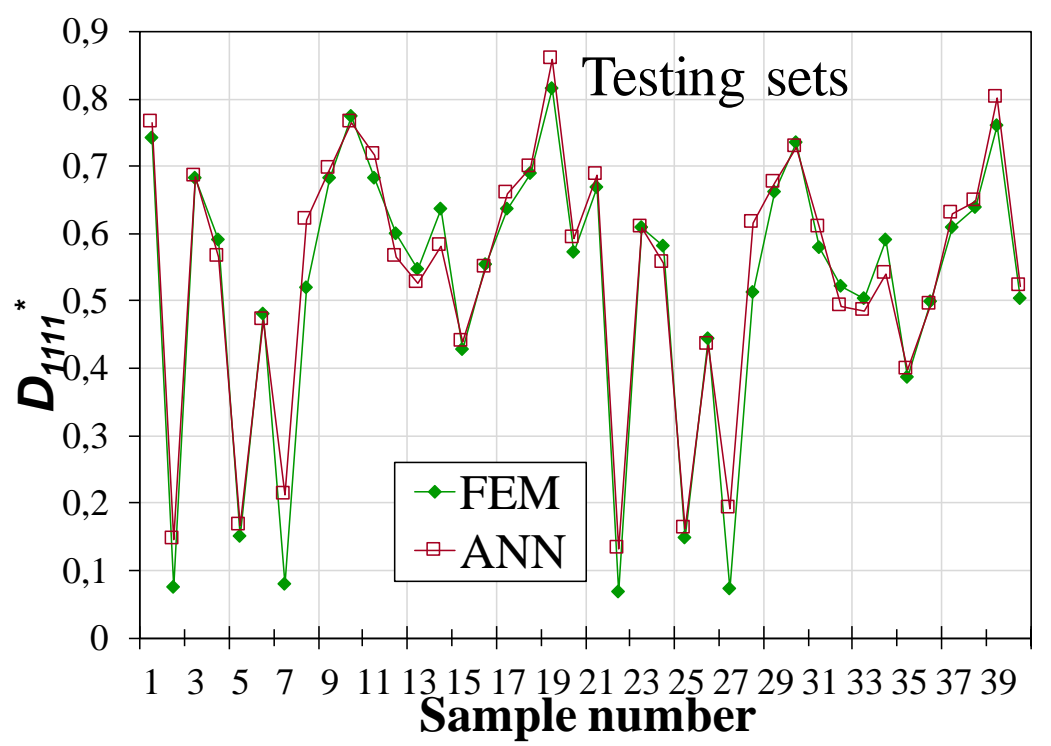

(b)

Fig.15. Evaluation of numerical and predicted coefficient by ANN for testing phase: (a) Normalized membrane elastic constant; (b) Normalized flexural elastic constant 


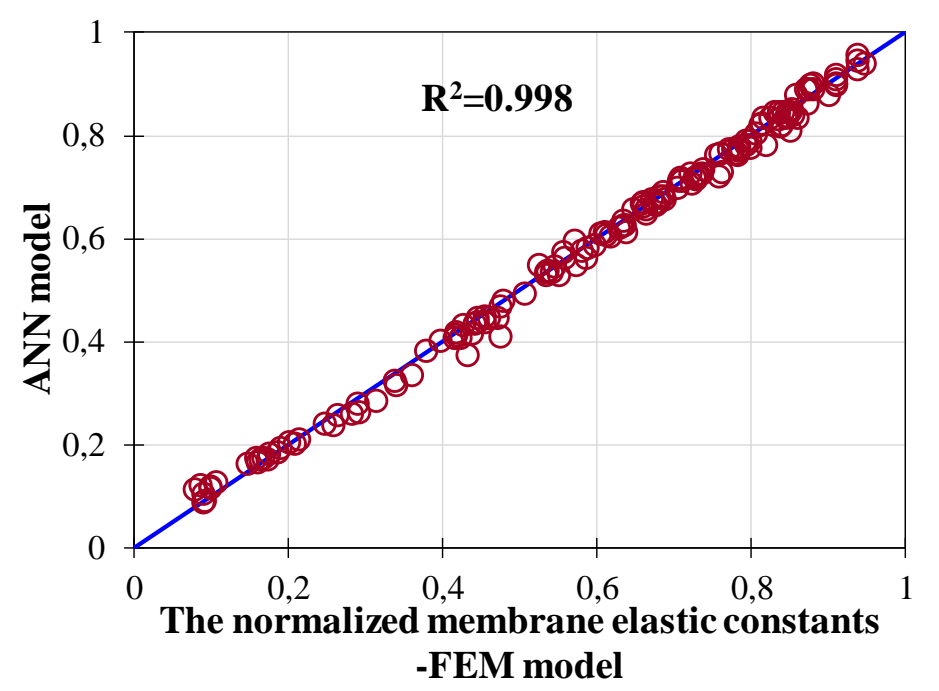

(a)

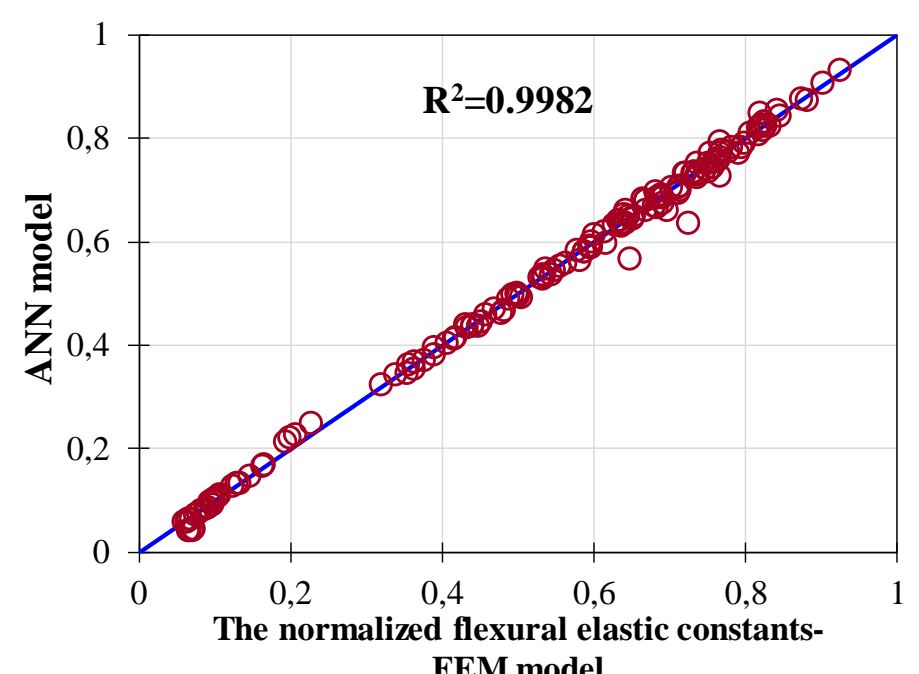

(b)

Fig.16. Comparison of ANN output with numerical values for training phase: (a) Normalized $A_{1111}$; (b) Normalized $D_{1111}$ 


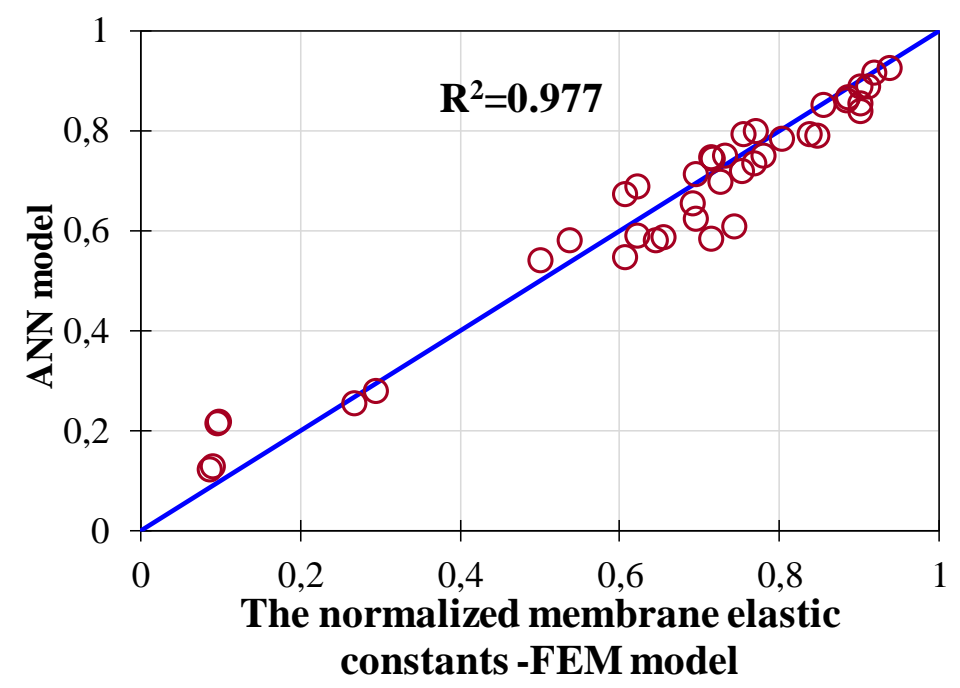

(a)

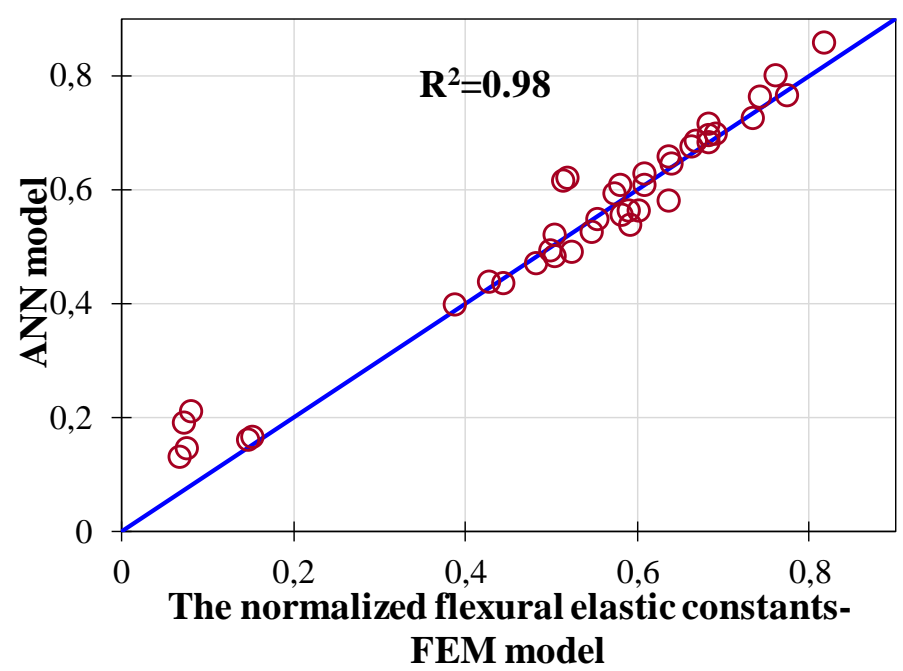

(b)

Fig.17. Comparison of ANN output with numerical values for testing phase: (a) Normalized $A_{1111}$; (b) Normalized $D_{1111}$ 


\section{Table captions}

Table 1. Chosen unit-cells geometrical properties

Table 2. Initial mechanical elastic properties of blocks and mortar

Table 3. Homogenized coefficients

Table 4. Frobenius of the constitute homogenized plates tensors for different bond pattern

Table 5. Factors and levels for factorial design

Table 6. Coefficients of determination of normalized responses 
Table 1. Chosen unit-cells geometrical properties

\begin{tabular}{llccc}
\hline Description & Geometrical parameters & $\begin{array}{c}\text { Running bond } \\
\text { value }(\mathrm{mm})\end{array}$ & $\begin{array}{c}\text { Stack bond } \\
\text { value }(\mathrm{mm})\end{array}$ & $\begin{array}{c}\text { English bond } \\
\text { value }(\mathrm{mm})\end{array}$ \\
\hline Length & $b+e^{v}$ & 510 & 510 & 510 \\
Height & $a^{\prime}+e^{h}$ & 410 & 210 & 420 \\
Thickness & $t$ & 200 & 200 & 410 \\
Mortar thickness & $e^{v}=e^{h}$ & 10 & 10 & 10 \\
Concrete block width & $c$ & 15 & 15 & 15 \\
\hline
\end{tabular}

Table 2. Initial mechanical elastic properties of blocks and mortar

\begin{tabular}{lcc}
\hline \multicolumn{1}{c}{ Variables } & Designation & Value \\
\hline Block Young modulus & $E^{B}$ & $44(\mathrm{GPa})$ \\
Mortar Young modulus & $E^{M}$ & $22(\mathrm{GPa})$ \\
Block Poisson ratio & $v_{b}$ & 0.25 \\
Mortar Poisson ratio & $v_{m}$ & 0.25 \\
\hline
\end{tabular}

Table 3. Homogenized coefficients

\begin{tabular}{|c|c|c|c|c|c|c|c|c|}
\hline $\begin{array}{l}\text { Homogenized } \\
\text { coefficients }\end{array}$ & $\begin{array}{l}A_{1111} \\
(\mathrm{~N} / \mathrm{mm})\end{array}$ & $\begin{array}{l}\boldsymbol{A}_{2222} \\
(\mathrm{~N} / \mathrm{mm})\end{array}$ & $\begin{array}{l}A_{1122} \\
(\mathrm{~N} / \mathrm{mm})\end{array}$ & $\begin{array}{l}A_{1212} \\
(\mathrm{~N} / \mathrm{mm})\end{array}$ & $\begin{array}{l}\boldsymbol{D}_{1111} \\
(\mathrm{~N} . \mathrm{mm})\end{array}$ & $\begin{array}{l}\boldsymbol{D}_{2222} \\
\text { (N.mm) }\end{array}$ & $\begin{array}{l}\boldsymbol{D}_{1122} \\
\text { (N.mm) }\end{array}$ & $\begin{array}{l}\boldsymbol{D}_{1212} \\
\text { (N.mm) }\end{array}$ \\
\hline $\begin{array}{l}\text { Running bond } \\
\text { value }\end{array}$ & $7.49 \times 10^{5}$ & $6.98 \times 10^{5}$ & $0.34 \times 10^{5}$ & $1.74 \times 10^{5}$ & $1.92 \times 10^{9}$ & $1.82 \times 10^{9}$ & $0.1 \times 10^{9}$ & $0.57 \times 10^{9}$ \\
\hline Stack bond value & $7.016 \times 10^{5}$ & $7.23 \times 10^{5}$ & $0.3 \times 10^{5}$ & $1.58 \times 10^{5}$ & $1.81 \times 10^{9}$ & $1.895 \times 10^{9}$ & $0.089 \times 10^{9}$ & $0.513 \times 10^{9}$ \\
\hline $\begin{array}{l}\text { English bond } \\
\text { value }\end{array}$ & $7.32 \times 10^{5}$ & $6.82 \times 10^{5}$ & $0.325 \times 10^{5}$ & $1.66 \times 10^{5}$ & $1.89 \times 10^{9}$ & $1.72 \times 10^{9}$ & $0.096 \times 10^{9}$ & $0.54 \times 10^{9}$ \\
\hline
\end{tabular}


Table 4. Frobenius of the constitute homogenized plates tensors for different bond pattern

\begin{tabular}{lcc}
\hline $\begin{array}{l}\text { Homogenized } \\
\text { coefficients }\end{array}$ & $\|A\|$ & $\|D\|$ \\
\hline Running bond value & $10.39 \times 10^{5}$ & $2.7 \times 10^{9}$ \\
\hline Stack bond value & $10.2 \times 10^{5}$ & $2.67 \times 10^{9}$ \\
\hline English bond value & $10.146 \times 10^{5}$ & $2.613 \times 10^{9}$ \\
\hline
\end{tabular}

Table 5. Factors and levels for factorial design

\begin{tabular}{cllll}
\hline Numerical factors & Level 1 & Level 2 & Level 3 & Level 4 \\
\hline Ratio $\xi=E^{B} / E^{M}$ & 1 & 5 & 10 & 30 \\
Thickness of mortar $e^{h}=e^{v}(\mathrm{~mm})$ & 5 & 10 & 20 & 30 \\
Ratio $a / b$ & 0.4 & 0.5 & 0.6 & - \\
Poisson ratios $v$ & 0.15 & 0.25 & - & - \\
Masonry texture & Running Bond (RB) & Stack bond (SB) & - \\
\hline
\end{tabular}


Table 6. Coefficients of determination of normalized responses

\begin{tabular}{|c|c|c|c|c|c|c|}
\hline \multirow{2}{*}{$\begin{array}{l}\text { Normalized } \\
\text { Homogenized Love- } \\
\text { Kirchhoff constants }\end{array}$} & \multicolumn{2}{|c|}{$\overline{R^{2}}$} & \multicolumn{2}{|c|}{$R M S E$} & \multicolumn{2}{|c|}{ MAPE (\%) } \\
\hline & Training & Testing & Training & Testing & Training & Testing \\
\hline$A_{1111}{ }^{*}$ & 0.998 & 0.977 & 0.012 & 0.041 & 3.191 & 4.1 \\
\hline$A_{2222}{ }^{*}$ & 0.9981 & 0.982 & 0.01 & 0.058 & 2.19 & 3.21 \\
\hline$A_{1122}{ }^{*}$ & 0.9972 & 0.971 & 0.0038 & 0.01 & 2.86 & 3.73 \\
\hline$A_{1212}{ }^{*}$ & 0.997 & 0.965 & 0.0076 & 0.023 & 3.82 & 4.51 \\
\hline$D_{1111}{ }^{*}$ & 0.9982 & 0.98 & 0.0137 & 0.045 & 2.93 & 3.94 \\
\hline$D_{2222}{ }^{*}$ & 0.9981 & 0.973 & 0.009 & 0.043 & 2.15 & 3.11 \\
\hline$D_{1122}{ }^{*}$ & 0.996 & 0.947 & 0.0045 & 0.013 & 4.62 & 5.31 \\
\hline$D_{1212}{ }^{*}$ & 0.9956 & 0.94 & 0.0076 & 0.023 & 4.7 & 5.41 \\
\hline
\end{tabular}

